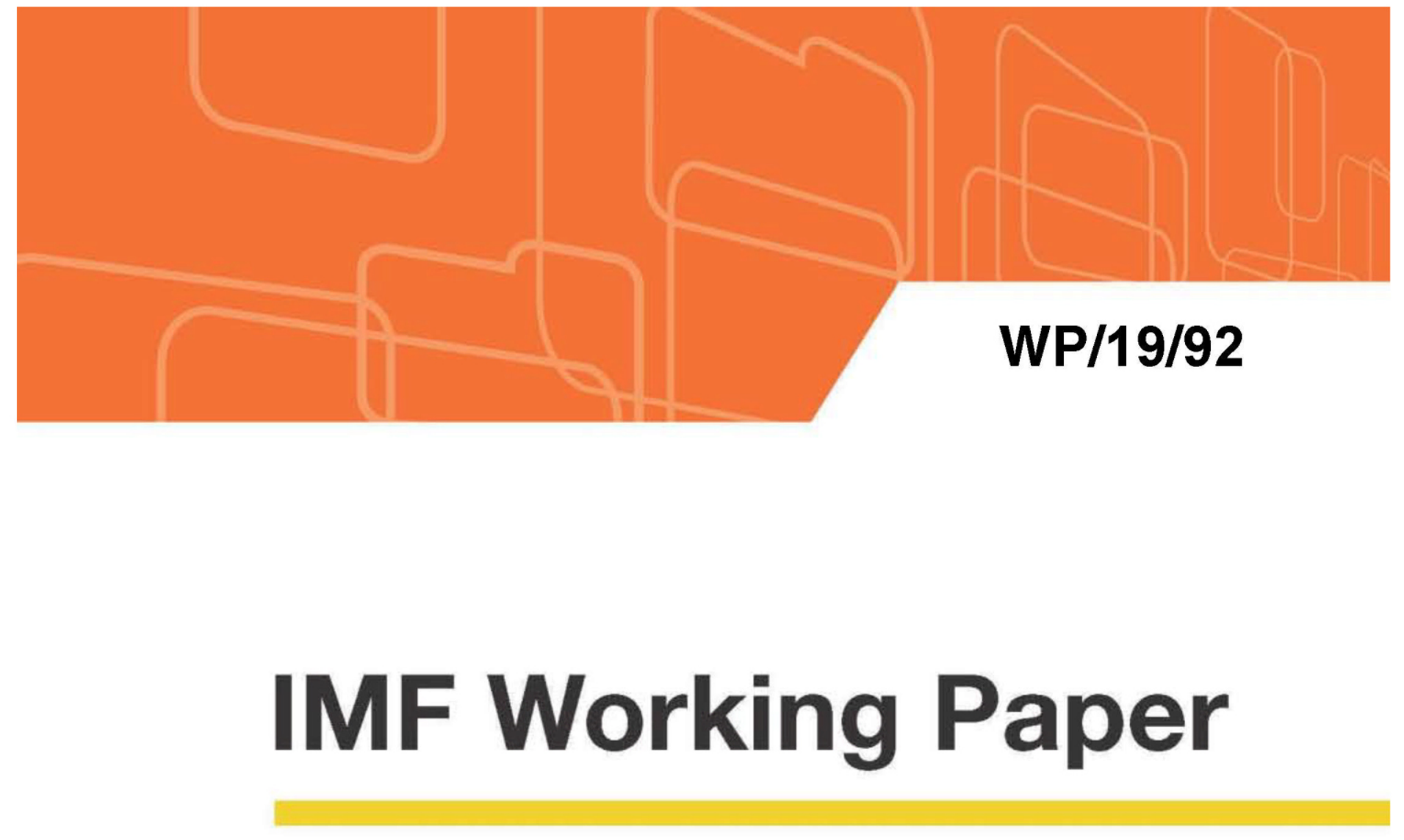

\title{
Inefficient Fire-Sales in Decentralized Asset Markets
}

by Ehsan Ebrahimy

IMF Working Papers describe research in progress by the author(s) and are published to elicit comments and to encourage debate. The views expressed in IMF Working Papers are those of the author(s) and do not necessarily represent the views of the IMF, its Executive Board, or IMF management. 


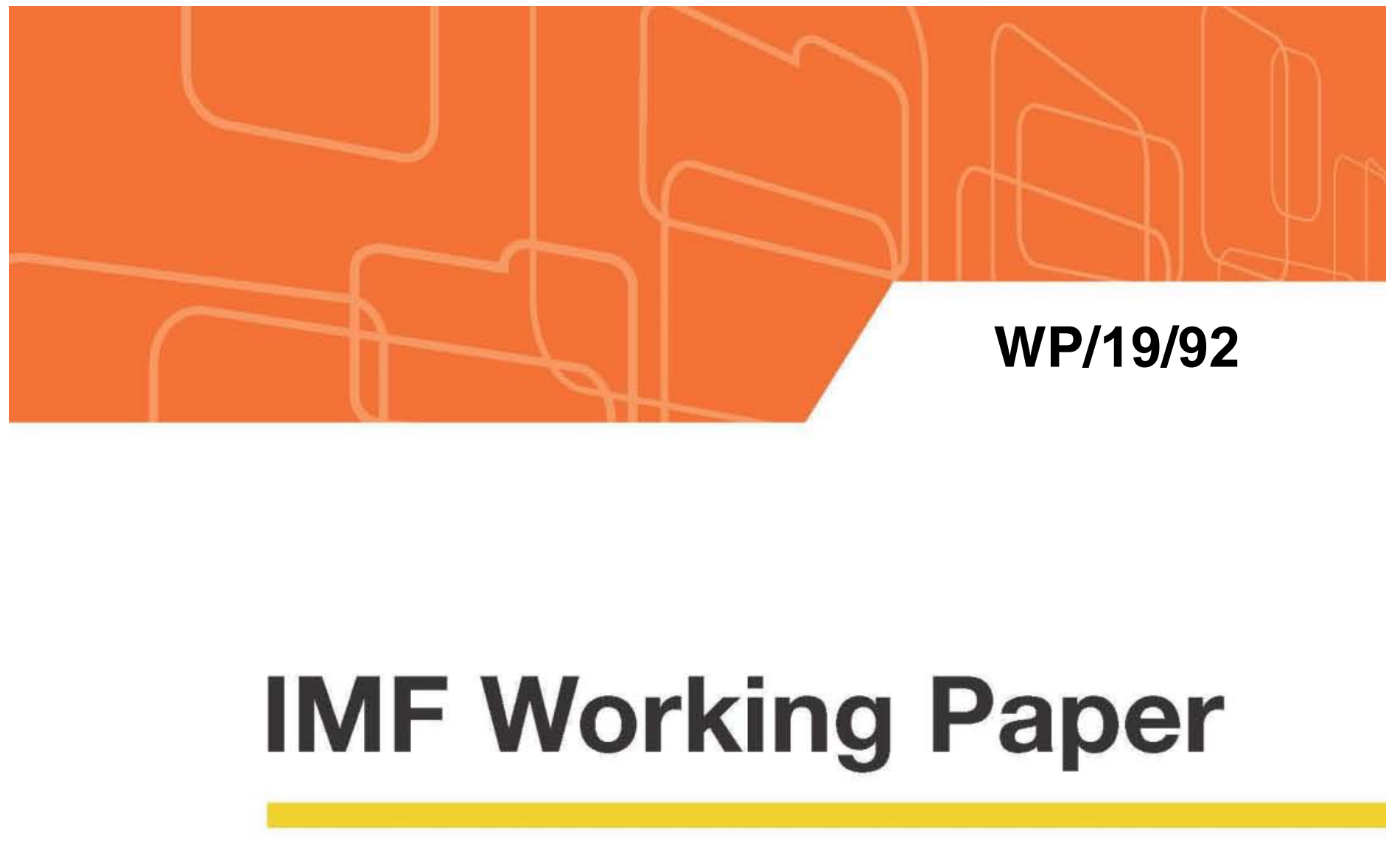

\section{Inefficient Fire-Sales in Decentralized Asset Markets}

by Ehsan Ebrahimy

IMF Working Papers describe research in progress by the author(s) and are published to elicit comments and to encourage debate. The views expressed in IMF Working Papers are those of the author(s) and do not necessarily represent the views of the IMF, its Executive Board, or IMF management.

I N TER N A T I O N A L MONETARY FUND 


\title{
IMF Working Paper
}

Research Department

\section{Inefficient Fire-Sales in Decentralized Asset Markets ${ }^{1}$}

Prepared by Ehsan Ebrahimy

Authorized for distribution by Maria Soledad Martinez Peria

May 2019

\section{IMF Working Papers describe research in progress by the author(s) and are published to elicit comments and to encourage debate. The views expressed in IMF Working Papers are those of the author(s) and do not necessarily represent the views of the IMF, its Executive Board, or IMF management.}

\begin{abstract}
Classic models of fire-sales that emphasize liquidity-constrained natural buyers can-not fully account for the asset fire-sales during the Financial Crisis of 2008. I present a model to demonstrate that fire-sales may happen even when there is a sizable pool of natural buyers and in the absence of asymmetric information, due to a coordina-tion failure among buyers. In particular, I show that when trade is decentralized and participation is endogenous, constrained asset demand and liquidity needs that are ex-pected to increase over time create complementarity among buyers' decisions to wait. This complementarity makes competitive markets prone to coordination failures and fire-sales which may be inefficient. I also discuss various policy options to eliminate the risk of fire-sales in such a setup.
\end{abstract}

JEL Classification Numbers: G01, G12, D61, D62, D83, E44

Keywords: fire-sales, coordination failure, decentralized markets, competitive search

Author’s E-Mail Address: EEbrahimy@imf.org

\footnotetext{
${ }^{1}$ I thank seminar participants in the Institute for Capacity Development, IMF Research Department, Bank of Finland/ESRB Conference on Systemic Risk Analytics and Lisbon Meeting on Game Theory and Applications. I thank Giovanni Dell'Ariccia, Anil Ari, Darrell Duffie, Divya Kirti, Romain Ranciere, Felix Vardy and especially Ruchir Agarwal for helpful comments and conversations. I also thank Jing Lu for excellent research assistance. All errors are my own.
} 


\section{Contents}

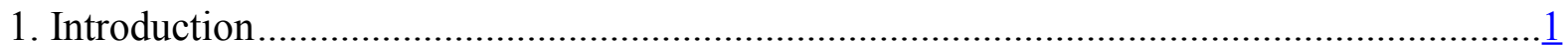

1.1 Related Literature.........................................................................

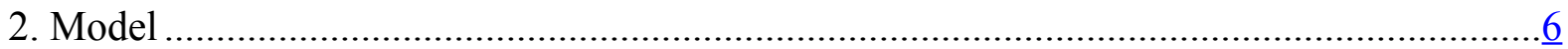

2.1 Competitive Search Equilibrium........................................................

2.2 Fragility and Fire-Sale .................................................................. 12

2.2.1 Conditions for Fragility.........................................................

2.2.2 Price Impact of Fire-Sale .......................................................

2.2 Welfare Analysis..............................................................................

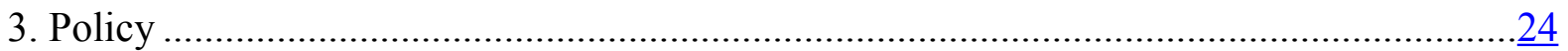

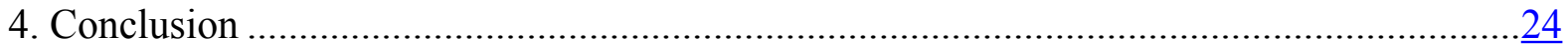

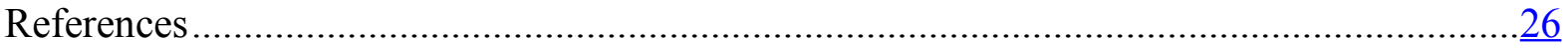

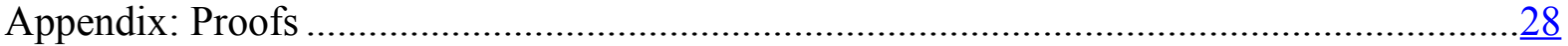

Figures

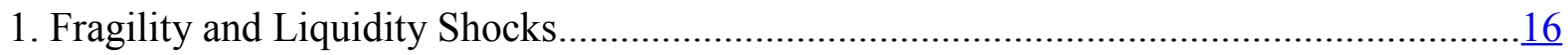

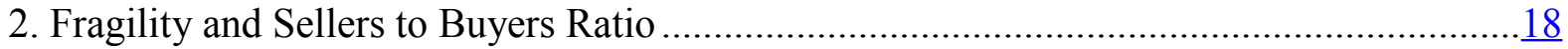

3. Price in Normal and Fire-Sale Equilibria........................................................................ 


\section{Introduction}

Sale of assets such as mortgage backed securities (MBS) at dislocated prices with deep discounts is a prominent feature in most narratives of the Financial Crisis of 2008. Any explanation of fire-sales should incorporate at least three broad facts about the asset markets during the crisis period. First, the initial shock to asset values seems to have been relatively small. Second, in conventional narratives of asset fire-sales sellers dump their holdings in the market (for example to deleverage) which should increase the trade volume. By contrast, the collapse of asset prices in many security markets during the recent crisis was accompanied by lower trade volume, akin to a market freeze. ${ }^{1}$ Finally, all financial assets subject to fire-sales during the crisis were traded in decentralized markets.

I show that when trade is decentralized, participation is endogenous, potential asset demand is constrained and liquidity needs are expected to increase over time, competitive markets are prone to coordination failure which results in fire-sale prices. Coordination failure and multiple equilibria stem from complementarity among buyers' (sellers') decisions to wait (to sell): when the potential demand of the asset is higher (lower) than its potential supply, a buyer's (seller's) decision to wait (to sell) can raise other buyers' (sellers') reservation utility by improving their future trade opportunities, thereby encouraging them to wait (sell) as well. Fire-sale is accompanied by lower trade volume due to buyers postponing trade when the potential asset demand is higher than the potential supply. In this case, fire-sale has a bigger price impact when markets are initially more liquid. Fragility and fire-sale require a medium degree of imbalance between buyers and sellers and a medium degree of market liquidity. Importantly fire-sales can be dominated in terms of welfare by the equilibrium with higher asset price and hence can be inefficient.

The possibility of coordination failure among buyers (or sellers) is a novel explanation for depressed asset prices during crises periods and maybe particularly relevant for understanding the type of fire-sales experienced during the Financial Crisis of 2008. The classic explanations of fire-sales emphasize liquidity constrained natural buyers who can either operate the asset productively (Shleifer and Vishny (1992)) or can better understand the asset' cash flows (Shleifer and Vishny (1997)). In contrast to these narratives, the focus of this paper is to demonstrate that fire-sale may happen even when there is a sizable pool of natural buyers of the asset and in the absence of any asymmetric information, due to strategic complementarity between buyers' decision to wait (or sellers' decision to sell). ${ }^{2}$ Importantly,

\footnotetext{
${ }^{1}$ See Park (2011) and Chernenko et al. (2014) for the documentation of these facts respectively.

${ }^{2} \mathrm{He}$, Khang, and Krishnamurthy (2010) show that there has been a sizable redistribution of asset backed securities (ABS) and leverage across the financial institutions rather than a uniform deleveraging in the banking system during the recent crisis, suggesting that there were less constrained buyers in the market.
} 
this type of coordination failure is possible only when markets are decentralized and already distressed: markets are hit by liquidity shocks and are subject to slow-moving capital (Duffie (2010)). This strategic waiting is a new amplification mechanism which is complementary to other explanations of slow-moving capital based on fundamentals of the market such as search frictions, asymmetric information or leverage.

A brief description of the model is as follows. There are two types of agents: sellers and buyers. Both types can consume over three periods but sellers are assumed to have a higher propensity to consume in the first two periods because they are hit by liquidity shocks. In this setup, one can think of the sellers as banks or other financial entities that have liquidity needs because they need to pay their short-term debts or meet a tighter regulatory capital requirements by liquidating assets in the market. Sellers hold one unit of an indivisible asset at the beginning while buyers can buy at most one unit of the asset. The number of buyers and sellers should be seen as proxies for the potential demand and supply in the market. Importantly participation by both sellers and buyers is endogenous (yet costless): they can trade at the initial date or postpone trade to the second period. Limited capacity of buyers may be due to tightening borrowing constraints in the financial market during a period of financial distress. Buyers post prices in the submarkets and sellers choose the the submarket and the corresponding price to maximize their utility. Sellers can choose a higher price but at the cost of facing a lower probability of trade as higher prices attract more sellers.

There are two types of equilibria which I label delayed and run equilibria. A delayed equilibrium is an equilibrium in which buyers or sellers are indifferent between participating or not participating in the market in the first period. As a result some buyers and sellers end up waiting until the second period for trade. A run equilibrium, on the other hand, is an equilibrium in which all buyers and sellers try to buy or sell in the first period.

The market is said to be fragile, where the two types of equilibria namely run and delayed coexist. When the market is fragile, the fire-sale equilibrium is defined as the equilibrium with the lower asset price. That is, a fire-sale equilibrium may be of either type: asset price falls either because (a) sellers run to sell immediately as they don't expect to find many opportunities to trade later or (b) buyers delay their purchase in the hope of finding even more desperate sellers in the future. If the number of buyers is more (less) than the sellers in the market, fire-sale happens in the form of a delayed (run) equilibrium. There are four critical elements making an asset market fragile in the model: a decentralized (yet competitive) asset market with agents deciding when to enter the market, liquidity shocks at

Whether these less constrained buyers should be considered "natural buyers" is an open empirical question. Adverse selection can be another reason why many buyers did not step in. Dow and Han (2018) show how asymmetric information may have increased during the crisis as specialized investors were capital constrained and hence prices became less informative. 
current and future dates which are increasing in magnitude over time and a medium degree of imbalance between potential supply and demand for the asset as well as a medium degree of market liquidity. I show that the equilibrium is unique in the absence of any of these elements.

In a centralized market, where trade takes place with certainty, the effect of each agent's decision to enter the market on others is fully priced and there are no non-priced externalities. In contrast, agent's decision to participate in a decentralized (yet competitive) market affects the probability of trade at current and future dates. A competitive market can price at most one of the two margins but not both. This leaves room for the presence of non-priced externalities and coordination failure which is at the heart of market fragility and fire-sales in this model.

To better understand these externalities and without loss of generality, consider the case where there are more buyers than sellers. In this case, a buyer's decision to wait decreases the ratio of buyers to sellers in the first period, and increases the ratio of buyers to sellers in the future period, thereby reducing market tightness in both current and future periods (relative to when the buyer decides to enter in the first period). ${ }^{3}$ The reduction in market tightness in both current and future periods, discourage and encourage other buyers to wait respectively. If the increase in probability of trade in the future date or equivalently the increase in current reservation utility of waiting is strong enough other buyers will decide to wait as well. In other words, there will be complementarity among buyers' waiting decisions. ${ }^{4}$

The fire-sale equilibrium is often inferior to the equilibrium with the higher asset price in terms of welfare due to the presence of non-priced externalities. More specifically, agents don't take into account the externality of their participation decisions on others' reservation utility at the initial date. When there are less sellers than buyers and there are multiple equilibria, the high asset price equilibrium ( run) dominates the fire-sale (delayed) equilibrium as long as the ratio of sellers to buyers is not too low. This means that the fire-sale equilibrium generates a lower aggregate trade surplus than the other equilibrium. A social planner can create an allocation which is Pareto superior to the fire-sale equilibrium by making lump sum transfers in the high asset price equilibrium. Therefore, the fire-sale equilibrium is inefficient in this case. When there are less buyers than sellers, on the other hand, the fire-sale equilibrium is always dominated by the high asset price equilibrium in terms of welfare.

\footnotetext{
${ }^{3}$ Market tightness is likely to decrease in the second period because the volume of trade, i.e., number of matches, decreases in the initial period. The ratio of remaining buyers to remaining sellers increases as the number of sellers are higher in the market.

${ }^{4}$ Note that in a delayed equilibrium this complementarity raises the gains from waiting just enough to make agents indifferent between participating in the current and future periods.
} 
I explore the model implications for the effect of fire-sales on price and trade volume in the market. When there are multiple equilibria, fire-sale prices coincide with lower (higher) volume of trade when there are less (more) sellers than buyers in the market. In other words, fire-sales are accompanied by a surge in the volume of trade in the market (a run equilibrium) only if there are too many sellers relative to buyers. In contrast, when there are too few sellers, fire-sales follow a decline in trade volume (a delayed equilibrium). This is an empirically testable prediction while noting that the number of buyers and sellers should be interpreted as the relative size of potential demand to potential supply. Moreover, I show that when the fire-sale happens in a delayed equilibrium the price impact of fire-sale, defined as the percentage decline in the asset price compared to the high asset price equilibrium, is bigger when markets are initially more liquid.

In my model, different types of policies may have different effects on market fragility. Asset purchases by the government in order to keep asset prices above a certain threshold or even a credible commitment to do so during the crisis can eliminate the fire-sale equilibria. Policies that change the market structure or the trading platform, akin to standardization of securities, introduction of Central Counterparty Clearing House (CCP) or requiring higher transparency, on the other hand, have ambiguous effects on market fragility. The potential effects of this latter set of policies depend on their quantitative effect on liquidity and demand in the market.

The rest of the paper is organized as follows. After literature review in section 1, the model is discussed in details in section 2.1. The conditions for fragility and the welfare analysis are discussed in 2.2 and 2.3 respectively. Section 3 discusses the effects of different type of policies and section 4 concludes.

\subsection{Related Literature}

There is a large literature on fire-sales going back to the classical paper Shleifer and Vishny (1992). In Shleifer and Vishny (1992) fire-sales happen because agents who are experts in using the asset are liquidity constrained. Shleifer and Vishny (1997), on the other hand, emphasizes limited arbitrage capital by specialized investors who understand the asset. But the explanation based on liquidity constrained experts is less applicable to financial assets. Moreover, not all buyers which may be considered experts/specialized investors, e.g., banks, were liquidity constrained during the crisis (He, Khang, and Krishnamurthy (2010)). As for the limited arbitrage capital, there were non-specialized investors with abundant resources

(e.g. Warren Buffet) to buy these assets and its not clear why these investors did not step in. 
Increasing information frictions can be one reason why outside investors did not buy distressed assets during the crisis. Dow and Han (2018) build a model to show how asymmetric information problems may have increased during the crisis leading to low demand by less liquidity constrained investors and hence depressed asset prices. In their model when specialized investors become liquidity constrained, market price becomes less informative about the fundamentals of the asset. This exacerbates the adverse selection problem by decreasing the supply of high quality assets. Increasing adverse selection lowers the valuations by nonspecialized investors and depresses the price.

Guerrieri and Shimer (2014) is a model of fire-sale of assets in decentralized markets with competitive search. Key features are competitive decentralized market and the presence of private information about the quality of asset. There is a unique equilibrium in which sellers signal quality of their asset by waiting longer for trade to take place. Fire-sale occurs when the distribution of asset quality worsens. Moreover, fire-sales are accompanied by illiquidity in the market. Chang (2018) studies a similar environment but with two dimensional private information. In her model sellers have private information about both asset quality and the degree of their distress. This implies that fire-sale happens only when trading volume is high and distressed sellers are willing to sell their assets at a steep discount in a semi-pooling equilibrium. In contrast to these papers, adverse selection doesn't play any role in this paper. Nonetheless, the model features fire-sale equilibria with both liquid/high-volume and illiquid/low-volume markets depending on the ratio of potential sellers to buyers.

In contrast to narratives based on adverse selection, market freezes happen in Diamond and Rajan (2011) due a debt overhang problem. Financial intermediaries refuse asset sales which can raise the chances of of their survival because much of the benefits in the case of survival are not accrued to shareholders.

This paper is also related to models of multiple equilibria in OTC markets. Vayanos and Weill (2008) use a model of decentralized asset markets with multiple equilibria to explain why newly issued treasury bonds are sold at a premium relative to the previously issued bonds. In their model, short sellers borrow newly issued bonds which endogenously makes them more liquid and special and hence they are sold at a premium. Such self-fulfilling equilibria exist due to the presence of search externalities: each agent's decision to borrow the newly issued bond enhances the liquidity and specialness of the bond, making others more willing to borrow the same type of bonds.

Guerrieri (2010) is a model of competitive search in the labor market with private information and limited commitment on the side of workers. Unlike typical models of directed search, the equilibrium is inefficient outside the steady state. The reason is that firms offering contracts at a given point in time do not internalize their externality on workers' outside 
option in previous periods. Similar to Guerrieri (2010), an intertemporal externality is the source of inefficiency and multiplicity in this model. However, the externality is from the past actions on future probability of trade.

Hanson and Sunderam (2013) look at the drivers of investors' demand for mortgage backed securities before and during the subprime crisis. They document that the trading for nontraditional securitizations declined significantly through the boom 2003-2007 and was extremely low during 2007-2009 bust. This in their view is consistent with buyers' strike narrative (as opposed to the fire-sale narrative), which emphasizes market freezes due to adverse selection or other frictions. This narrative is more consistent with falling prices accompanied by lower volume of trade. They also find that investors sold more of their liquid securities, e.g., government-guaranteed MBS, during the crisis.

Boudoukh et al. (2016) document puzzling behavior of newly issued sovereign bonds spread during periods of financial distress. They find that the newly issued sovereign bonds, which are more liquid and more expensive during normal times, become cheaper in time of crises specially in the case of low quality sovereigns. In this paper, the price impact of fire-sale as well as the drop in trade volume are larger for more liquid assets when fire-sale happens in a delayed equilibrium. The findings in Boudoukh et al. (2016) would be consistent with these predictions if trade volume in newly issued sovereigns decline more significantly than the old bonds.

This paper is also related to the large body of research on multiple equilibria in currency markets. Obstfeld (1996) provides a review of the relevant models. Unlike this model, models of currency attacks feature centralized markets. The key ingredient which makes the centralized market vulnerable to bad equilibria with large currency devaluations is the presence of a non-negligible strategic agent namely the government.

Bernardo and Welch (2004) is an example of a model of fire-sale and run in a stock market. Risk neutral investors fear that they need to liquidate shares after a run takes place and before prices recover. This fear may force investors to sell today and may cause the run itself. Similar to this model, future liquidity shocks play a key role in causing a run today.

\section{Model}

There are three periods $t=0,1,2$ and two types of agents, measure 1 of buyers and $m>0$ of sellers, who can trade an indivisible asset in the first two periods. The asset pays off its only dividend $d_{2}>0$ units of consumption goods at $t=2$. Agents preferences are as follows: 


$$
\left\{\begin{array}{l}
\boldsymbol{U}_{S}=\mathbb{E}_{0}\left\{\delta_{0} C_{0}+\delta_{1} C_{1}+C_{2}\right\}, \\
\boldsymbol{U}_{B}=\mathbb{E}_{0}\left\{C_{0}+C_{1}+C_{2}\right\},
\end{array}\right.
$$

Where subscripts $S$ and $B$ indicate the seller and buyer. We assume that buyers have big enough endowments in $t=0,1$ to pay for the asset. ${ }^{5}$ Both buyers and sellers can save at the (gross) real rate of one in $t=0,1$. Timing of events are as follows. In each period, both types of agents first decide how much to save, then if they want to participate in the market and finally consume whatever has not been saved or spent on market transactions. ${ }^{6}$

Coefficients $\delta_{0}$ and $\delta_{1}$ are seller's marginal utility of consumption in periods $t=0,1$. They capture a liquidity shock today or an expected liquidity shock tomorrow respectively. Sellers in this model may represent banks or other financial entities that need to pay their short term debts or meet a tighter regulatory capital requirements by liquidating assets in the market. Therefore we make the following assumption:

Assumption $1 . \delta_{0}>1$ and $\delta_{1}>1$.

Sellers hold a unit of the asset in $t=0$ and buyers may buy at most one unit of the asset in either $t=0,1$. Note that given Assumption 1, buyers (sellers) have no incentives to sell (buy) if they purchase the asset in the market at $t=0$.

Trade can take place in a decentralized market in both $t=0,1$ with competitive search and random matching. ${ }^{7}$ In each period, buyers post prices and form submarkets each of which represent the subset of buyers who have posted the same price. Sellers of the asset observe these prices and choose the submarket and the corresponding price to maximize their utility. The buyers' problem at $t=1$ if she participates is:

$$
\left\{\begin{array}{l}
V_{1}^{B}=\max _{\sigma_{1}, p_{1}} q_{1}^{B}\left(\sigma_{1}\right)\left(d_{2}-p_{1}\right) \\
\text { s.t. } \quad \bar{U}_{1}^{S} \leq q_{1}^{S}\left(\sigma_{1}\right) \delta_{1} p_{1}+\left(1-q_{1}^{S}\left(\sigma_{1}\right)\right) d_{2}
\end{array}\right.
$$

Buyer strictly prefers to participate in the market as long as $V_{1}^{B}>R_{1}^{B}$ where $R_{1}^{B}$ is the reservation utility of the buyer at $t=1$ and is equal to zero. If $V_{1}^{B}=R_{1}^{B}$ buyer is indifferent

\footnotetext{
${ }^{5}$ The value of sellers' endowment is irrelevant due to risk neutrality.

${ }^{6}$ The results are equivalent to assuming no saving. We can allow for saving after market transactions if we introduce risk aversion.

${ }^{7}$ The main results of the model hold in an environment with random search. Using competitive search shows that the complementarity among agents' participation decisions and the resulting multiplicity as well as the inefficiency results don't depend on inherent externalities in random search.
} 
between participating and staying out of the market. $\sigma_{1}$ is the ratio of sellers to buyers or the length of the queue for the submarket with posted price of $p_{1}$. Moreover, $q_{1}^{B}$ and $q_{1}^{S}$ are probabilities of being matched with a seller and buyer respectively in the submarket with the queue length of $\sigma_{1}$ which depends on the matching technology, i.e., market microstructure. The participation constraint by the seller in the problem above requires that the seller's utility from trade is no less than the maximum, $\bar{U}_{1}^{S}$, she can obtain outside the match where:

$$
\bar{U}_{1}^{S}=\max \left(V_{1}^{S}, R_{1}^{S}\right)
$$

Where $V_{1}^{S}$ and $R_{1}^{S}$ are sellers continuation utility from participating and reservation utility of not participating in the market at $t=1$. We note that $R_{1}^{S}=d_{2}$. Using $q_{1}^{B}=\sigma_{1} q_{1}^{S}$ and that the participation constraint is binding in equilibrium, one has:

$$
\left\{\begin{array}{l}
q_{1}^{S}\left(\sigma_{1}\right)\left(\delta_{1} p_{1}-d_{2}\right)=\bar{U}_{1}^{S}-d_{2} \Rightarrow \\
\delta_{1} q_{1}^{B}\left(\sigma_{1}\right)\left(d_{2}-p_{1}\right)=\sigma_{1}\left(\bar{U}_{1}^{S}-d_{2}\right)-\left(\delta_{1}-1\right) q_{1}^{B}\left(\sigma_{1}\right) d_{2}
\end{array}\right.
$$

Hence one can simplify the buyers problem:

$$
V_{1}^{B}=\max _{\sigma_{1}}\left\{\frac{1}{\delta_{1}}\left(\left(\delta_{1}-1\right) q_{1}^{B}\left(\sigma_{1}\right) d_{2}-\sigma_{1}\left(\bar{U}_{1}^{S}-d_{2}\right)\right)\right\}
$$

Similarly, the problem of buyers at $t=0$ is:

$$
\left\{\begin{array}{l}
V_{0}^{B}=\max _{\sigma_{0}, p_{0}}\left\{q_{0}^{B}\left(\sigma_{0}\right)\left(d_{2}-p_{0}\right)+\left(1-q_{0}^{B}\left(\sigma_{0}\right)\right) \bar{U}_{1}^{B}\right\} \\
\text { s.t. } \quad \bar{U}_{0}^{S} \leq q_{0}^{S}\left(\sigma_{0}\right) \delta_{0} p_{0}+\left(1-q_{0}^{S}\left(\sigma_{0}\right)\right) \bar{U}_{1}^{S}
\end{array}\right.
$$

Where $\bar{U}_{1}^{B}=\max \left(V_{1}^{B}, R_{1}^{B}\right)$ is the maximum utility for buyers if they wait until $t=1$. Note that given our notation this is the reservation utility at $t=0$ for buyers as well, i.e., $R_{0}^{B}=\bar{U}_{1}^{B}$. And $\bar{U}_{0}^{S}$ is the maximum utility sellers can obtain in the market at time zero. 


\subsection{Competitive Search Equilibrium}

I assume that for any measure $s$ of sellers and $b$ of buyers at any given period who are active in any of the submarkets, the number of matches is given by:

$$
\mathbb{M}(s, b)=\gamma s^{1-\alpha} b^{\alpha}
$$

where $0<\alpha<1$ and $0<\gamma<1$ is the efficiency of the matching function. Note that the market liquidity of the asset is affected by both $\gamma$ and $m$.

We restrict our parameters so that the implied probabilities will be always less than one: Assumption 2. $\gamma^{\frac{1}{\alpha}}<\frac{m-\gamma m^{1-\alpha}}{1-\gamma m^{1-\alpha}}<\gamma^{\frac{-1}{1-\alpha}}$.

To solve for the equilibrium we start at $t=1$. Using 3 , the first order conditions (FOC) for buyers at $t=1$ are:

$$
\left(\delta_{1}-1\right) \frac{d q_{1}^{B}\left(\sigma_{1}\right)}{d \sigma_{1}} d_{2}=\bar{U}_{1}^{S}-d_{2} \Rightarrow
$$

Given our matching technology 6 , we have $q_{1}^{B}\left(\sigma_{1}\right)=\gamma \sigma_{1}^{1-\alpha}, q_{1}^{S}\left(\sigma_{1}\right)=\gamma \sigma_{1}^{-\alpha}$ and hence using 7 and 2 we can obtain the unique equilibrium price at $t=1$ :

$$
\left\{\begin{array}{l}
(1-\alpha)\left(\delta_{1}-1\right) d_{2}=\delta_{1} p_{1}-d_{2} \Rightarrow \\
p_{1}^{*}=\frac{1+(1-\alpha)\left(\delta_{1}-1\right)}{\delta_{1}} d_{2}
\end{array}\right.
$$

Note that we have:

$$
\frac{d_{2}}{\delta_{1}}<p_{1}^{*}<d_{2}
$$

As $0<\alpha<1$. This implies that both buyers and sellers strictly prefer to participate in the market at $t=1$. In other words $\bar{U}_{1}^{S}=V_{1}^{S}>R_{1}^{S}$ and $\bar{U}_{1}^{B}=V_{1}^{B}>R_{1}^{B}$. Therefore, there is a unique equilibrium price in the market at $t=1$ with full participation of both buyers and sellers.

Using 2 and the equilibrium price 8, we can obtain the continuation utilities $\bar{U}_{1}^{S}$ and $\bar{U}_{1}^{B}$ (or equivalently $V_{1}^{S}$ and $V_{1}^{B}$ ) as follows: 


$$
\left\{\begin{array}{l}
\bar{U}_{1}^{S}=V_{1}^{S}=\left(1+(1-\alpha)\left(\delta_{1}-1\right) \gamma \sigma_{1}^{*-\alpha}\right) d_{2} \\
\bar{U}_{1}^{B}=V_{1}^{B}=\frac{\alpha\left(\delta_{1}-1\right)}{\delta_{1}} \gamma \sigma_{1}^{* 1-\alpha} d_{2}
\end{array}\right.
$$

Turning to $t=0$, we can use the participation constraint in 5 , which must hold with equality in equilibrium, to obtain:

$$
\left\{\begin{array}{l}
q_{0}^{S}\left(\sigma_{0}\right) \delta_{0} p_{0}=\sigma_{0}\left(\bar{U}_{0}^{S}-\left(1-q_{0}^{S}\left(\sigma_{0}\right)\right)\right) \Rightarrow \\
q_{0}^{B}\left(\sigma_{0}\right) p_{0}=\frac{\sigma_{0}}{\delta_{0}}\left(\bar{U}_{0}^{S}-\left(1-q_{0}^{S}\left(\sigma_{0}\right)\right)\right)
\end{array}\right.
$$

Since $q_{0}^{B}\left(\sigma_{0}\right)=\sigma_{0} q_{0}^{S}\left(\sigma_{0}\right)$. This simplifies the buyer's maximization at $t=0$ :

$$
V_{0}^{B}=\max _{\sigma_{0}}\left\{q_{0}^{B}\left(\sigma_{0}\right)\left(d_{2}-\frac{1}{\delta_{0}} \bar{U}_{1}^{S}-\bar{U}_{0}^{B}\right)-\frac{\bar{U}_{0}^{S}-\bar{U}_{1}^{S}}{\delta_{0}} \sigma_{0}+\bar{U}_{1}^{B}\right\}
$$

The FOC for an interior solution with full participation is:

$$
\left\{\begin{array}{l}
\left(d_{2}-\frac{1}{\delta_{0}} \bar{U}_{1}^{S}-\bar{U}_{0}^{B}\right) \frac{d q_{1}^{B}\left(\sigma_{0}\right)}{d \sigma_{0}}=\frac{\bar{U}_{0}^{S}-\bar{U}_{1}^{S}}{\delta_{0}} \Rightarrow \\
\sigma_{0}^{*-\alpha}=\frac{\bar{U}_{0}^{S}-\bar{U}_{1}^{S}}{\delta_{0} \gamma(1-\alpha)\left(d_{2}-\frac{1}{\delta_{0}} \bar{U}_{1}^{S}-\bar{U}_{0}^{B}\right)}
\end{array}\right.
$$

Full participation requires $d_{2}-\frac{1}{\delta_{0}} \bar{U}_{1}^{S}-\bar{U}_{0}^{B}>0$ and $\bar{U}_{0}^{S}-\bar{U}_{1}^{S}>0$. The price at $t=0$ can be obtained using participation constraint of the seller at $t=0$ and 12 :

$$
p_{0}^{*}=(1-\alpha)\left(d_{2}-\bar{U}_{1}^{B}\right)+\alpha \frac{1}{\delta_{0}} \bar{U}_{1}^{S}
$$

Where $\bar{U}_{1}^{B}$ and $\bar{U}_{1}^{S}$ are given by 9. Full participation equilibrium, however, is not the only possible outcome. Another type of equilibria may exist where agents are indifferent between participating or staying out of the market at $t=0$. This happens only when: 


$$
\left\{\begin{array}{l}
\bar{U}_{0}^{S}-\bar{U}_{1}^{S}=0 \\
d_{2}-\frac{1}{\delta_{0}} \bar{U}_{1}^{S}-\bar{U}_{0}^{B}=0
\end{array}\right.
$$

Equations 15 imply that buyers cannot raise their lifetime utility by changing the probability or the price they post in the market. The term $d_{2}-\frac{1}{\delta_{0}} \bar{U}_{1}^{S}-\bar{U}_{0}^{B}$ is equal to $d_{2}-\frac{1}{\delta_{0}} \bar{U}_{1}^{S}-V_{1}^{B}$ as $\bar{U}_{0}^{B}=V_{1}^{B}$. This latter expression is the total extra surplus of trade (conditional on trade taking place), a fraction $\alpha$ of which accrues to the buyer. It is evident from the first equation in 15 that for buyers to be indifferent, sellers must be indifferent as well. The second equation above is in terms of continuation utilities of sellers and buyers at $t=1$ which are given by 9 . We can use 9 to obtain the following equation in the inverse of market tightness at $t=1$ :

$$
\frac{1}{\delta_{0}}+\frac{(1-\alpha)\left(\delta_{1}-1\right)}{\delta_{0}} \gamma \sigma_{1}^{*-\alpha}+\frac{\alpha\left(\delta_{1}-1\right)}{\delta_{1}} \gamma \sigma_{1}^{* 1-\alpha}=1
$$

Any solution to 16 pins down the market tightness and continuation utilities at $t=1$ for an equilibrium where agents are indifferent between waiting and participating at time zero. We will examine later the conditions under which 16 admits a feasible solution. To save space, it is convenient to define the following function for future reference:

Definition 1. Function $f(\sigma)$ is defined as:

$$
f(\sigma) \equiv \frac{1}{\delta_{0}}+\frac{(1-\alpha)\left(\delta_{1}-1\right)}{\delta_{0}} \gamma \sigma_{1}^{-\alpha}+\frac{\alpha\left(\delta_{1}-1\right)}{\delta_{1}} \gamma \sigma_{1}^{1-\alpha},
$$

The market price will be given by the sellers' participation constraint in 5 combined with 15:

$$
\left\{\begin{array}{l}
p_{0}^{* *}=\frac{1}{\delta_{0}} \bar{U}_{1}^{S} \Rightarrow \\
p_{0}^{* *}=\left\{\frac{1}{\delta_{0}}+\frac{(1-\alpha)\left(\delta_{1}-1\right)}{\delta_{0}} \gamma \sigma_{1}^{*-\alpha}\right\} d_{2}
\end{array}\right.
$$

The following defines a competitive search equilibrium in the model: 
DEFINITION 2. An equilibrium is a set of prices, probabilities of trade in the market, (inverse of) market tightness, measures of sellers and buyers participating in the markets and utilities at $t=0,1$, denoted by $\left\{p_{t}^{*}, q_{t}^{* j}, \sigma_{t}^{*}, \mu_{t}^{* j}, V_{t}^{j}\right\}$ for $t=0,1$ and $j \in\{B, S\}$, such that $\sigma_{0}^{*}=\frac{\mu_{0}^{* S}}{\mu_{0}^{* B}}$ and $\sigma_{1}^{*}=\frac{\mu_{1}^{* S}}{\mu_{1}^{* B}}$ and sellers and buyers are maximizing their welfare according to 1, 3, 7, 5 and 12 and $\mu_{0}^{* S}=m$ if $V_{0}^{S}>R_{0}^{S}$ and $\mu_{0}^{* B}=1$ if $V_{0}^{B}>R_{0}^{B}$.

Before moving to the next section, I can define some of the notions which will be used throughout:

DeFinition 3. Given a set of parameters, $\left\{m, \gamma, \delta_{0}, \delta_{1}\right\}$, market is said to be fragile if both full participation and limited participation equilibria exist. When market is fragile we call the equilibrium with full and limited participation a $\underline{\text { run }}$ and delayed equilibrium denoted by superscripts $R$ and $D$ respectively. The strictly lower price at $t=0$ between the run and delayed equilibria $\left(p_{0}^{* D}\right.$ or $\left.p_{0}^{* R}\right)$ is called a fire-sale price.

\subsection{Fragility and Fire-Sale}

I have characterized competitive equilibria in the previous section. In this section, I examine the conditions under which markets are fragile and whether and when any fire-sale takes place.

First, we want to characterize the behavior of the price at $t=0$ for the delayed and run equilibria when we have fragility in the market. We restate the equilibrium price for the run equilibrium:

$$
\left\{\begin{array}{l}
p_{0}^{*}=(1-\alpha)\left(d_{2}-\bar{U}_{1}^{B}\right)+\alpha \frac{1}{\delta_{0}} \bar{U}_{1}^{S} \Rightarrow \\
p_{0}^{*}\left(\sigma_{1}^{*}\right)=\left\{(1-\alpha)\left(1-\frac{\alpha\left(\delta_{1}-1\right)}{\delta_{1}} \gamma \sigma_{1}^{* 1-\alpha}\right)+\frac{\alpha}{\delta_{0}}\left(1+(1-\alpha)\left(\delta_{1}-1\right) \gamma \sigma_{1}^{*-\alpha}\right)\right\} d_{2}
\end{array}\right.
$$

The above equation for the price at $t=0$ holds across different equilibria: for the delayed equilibrium the equation collapses to 18 as we have $d_{2}-\bar{U}_{1}^{B}=\frac{1}{\delta_{0}} \bar{U}_{1}^{S}$. Taking the derivative with respect to the (inverse of) market tightness gives:

$$
\frac{d p_{0}^{*}\left(\sigma_{1}^{*}\right)}{d \sigma_{1}^{*}}=\left(-\frac{\alpha}{\delta_{0}} \sigma_{1}^{*-(1+\alpha)}-\frac{1-\alpha}{\delta_{1}} \sigma_{1}^{*-\alpha}\right) d_{2}<0
$$

The equilibrium price at $t=0$ is decreasing in inverse of market tightness $\sigma_{1}^{*}$, because 
higher $\sigma_{1}^{*}$ makes finding buyers next period less likely and consequently reduces seller's continuation value or reservation utility. This in turn leads the sellers to accept lower prices in equilibrium at $t=0$.

The change in the price at $t=0$, when agents switch from one equilibrium to another, depends on the change in $\sigma_{1}^{*}$. As the equilibrium in the market at $t=1$ always entails full participation of buyers and sellers we have:

$$
\sigma_{1}^{*}=\frac{m-\nu_{0}}{1-\nu_{0}}
$$

Where $\nu_{0}$ is the total number of matches formed or the volume of trade in $t=0$. We note that $\frac{d \sigma_{1}^{*}}{d \nu}>0$ if and only if $m>1$. We know that full participation always has the highest volume of trade and therefore we can summarize the results as follows:

LEMMA 1. Whenever we have market fragility, the following hold for the delayed and run equilibria:

$$
p_{0}^{* R}<p_{0}^{* D} \Longleftrightarrow m>1
$$

Where superscripts $D$ and $R$ denote delayed and run equilibria. In other words, we have fire-sale either in a run equilibrium where $m>1$ or in a delayed equilibrium where $m<1$. Moreover, $\nu_{0}$ or the volume of trade at $t=0$ is higher when the price is higher if and only if $m<1$.

When there are more (less) buyers than sellers in the market, the delayed equilibrium has a lower (higher) price than the run as less participation in the market at $t=0$ lowers (raises) the continuation value of the sellers and therefore the current market price. The latter statement is true because by 21 , when $m<1(m>1)$ the resulting market tightness at $t=1$ is an increasing (decreasing) function of the volume of trade at $t=0$.

\subsubsection{Conditions for Fragility}

Before any further analysis, its helpful to take a look at a benchmark where trade takes place in a centralized competitive asset market:

LEMMA 2. (Centralized market as a benchmark) In a centralized competitive asset market, and except for the knife-edge case of $m=1$, the equilibrium prices at which trade takes place are unique. When $m>1$ the following are true. All trades take place in $t=0$ at $p_{0}=d_{2} / \delta_{0}$ 
if $\delta_{0}>\delta_{1}$ or in $t=1$ at $p_{1}=d_{2} / \delta_{1}$ if $\delta_{0}<\delta_{1}$. And $p_{0}=p_{1}=d_{2} / \delta_{0}=d_{2} / \delta_{1}$ and the volumes of trade in $t=0,1$ are indeterminate if $\delta_{0}=\delta_{1}$. In this case buyers enjoy all of the trade surplus. When $m<1$ all trades take place in $t=0$ at $p_{0}=d_{2}$ when $\delta_{1}<\delta_{0}$ and sellers enjoy all of trade surplus. When $\delta_{1}=\delta_{0}$ we have $p_{0}=d_{2}$ but the volume of trade at $t=0$ is indeterminate. Finally if $\delta_{1}>\delta_{0}$ no trade takes place at $t=0$ and asset is traded only at $t=1$.

The above lemma implies that market fragility within this setup is not a feature of centralized trading. This is because there are no externalities and no possibility of coordination failure among sellers or buyers. When sellers and buyers can trade with certainty, delaying trade by one agent at $t=0$ has no externality on others' decisions as it doesn't affect the probability of trade for other agents.

By contrast, in a decentralized market each agent's decision to participate at $t=0$ may change the probability of trade at both dates, $t=0,1$, for others. The following lemma establishes the existence of multiplicity at $t=0$ :

LEMmA 3. There are potentially two types of equilibria with strictly positive volume of trade at $t=0$ in the model. One type features a unique full participation or run equilibrium that is characterized by 8, 9, 12, 13 and the following additional conditions:

$$
\left\{\begin{array}{l}
V_{0}^{S}>R_{0}^{S}, \quad V_{0}^{B}>R_{0}^{B}, \quad \mu_{0}^{* S}=m, \quad \mu_{0}^{* B}=1 \\
\mu_{1}^{* S}=m-\gamma m^{1-\alpha}, \quad \mu_{1}^{* B}=1-\gamma m^{1-\alpha} \\
\sigma_{1}^{*}=\frac{m-\gamma m^{1-\alpha}}{1-\gamma m^{1-\alpha}}, \quad \sigma_{0}^{*}=m
\end{array}\right.
$$

In the second type or the limited participation or delayed equilibrium, sellers and buyers are indifferent between participating in the market and staying out of the market at $t=$ 0. Equilibrium prices and utilities at $t=0,1$, (inverse of) tightness measure and hence probabilities of finding a partner at $t=1$ are pinned down using 9, 8, 15, 16, 18 and the following additional conditions:

$$
\left\{\begin{array}{l}
\gamma \mu_{0}^{* S^{1-\alpha}} \mu_{0}^{* B^{\alpha}}=\frac{m-\sigma_{1}^{*}}{1-\sigma_{1}^{*}}, \quad \sigma_{0}^{*}=\frac{\mu_{0}^{* S}}{\mu_{0}^{* B}} \\
\mu_{1}^{* S}=m-\gamma \mu_{0}^{* S^{1-\alpha}} \mu_{0}^{* B^{\alpha}}, \quad \mu_{1}^{* B}=1-\gamma \mu_{0}^{* S^{1-\alpha}} \mu_{0}^{* B^{\alpha}}
\end{array}\right.
$$


Note that Lemma 3 characterizes equilibria with positive volume of trade at $t=0$. This is because for some parameter values, such as when $\frac{\delta_{1}}{\delta_{0}}$ or $m$ are too large, an equilibrium with no trade at time zero can exist. We can make an additional assumption to eliminate this possibility which leaves the rest of the analysis virtually intact ${ }^{8}$. But to reduce complexity, I just ignore the possibility of a no-trade equilibrium in the remainder of the paper.

Unlike random search, competitive search allows the intratemporal externalities of each agent's action to be fully priced in the market ${ }^{9}$. But the intertemporal externalities of agent's decision at $t=0$ on others agents' probability of trade at $t=1$ and hence their reservation utility at $t=0$ are not fully captured by price mechanism at $t=0$. This is the source of coordination problems and multiplicity in the model.

Consider for example, without any loss of generality, the case of a buyer at $t=0$. Her decision whether or not to enter the market at $t=0$ and what price to offer affects market tightness at both $t=0$ and $t=1$. The buyer offers contract in which she specifies the price while considering how that price will affects sellers' incentives and consequently the market tightness in her submarket at $t=0$. But the buyer takes others' continuation value of not trading at $t=0$ as a given and fails to incorporate the externality of her decision on market tightness at $t=1$ and hence the reservation utilities of others at $t=0$.

In order to have multiplicity, these externalities should lead to complementarity among agents' actions. Complementarity arises because a buyer's decision (a seller's decision) to wait (to sell) at $t=0$ when $m<1$ (when $m>1$ ) reduces market tightness at $t=1$, i.e., increases $\sigma_{1}^{*}$. This increases the reservation utility of other buyers (sellers) at $t=0$ which encourages them to wait (to sell) at $t=0$ as well.

We now turn to the conditions for the existence of multiple equilibria. To this end, we need to make the following assumptions:

Assumption 3. We have $f\left(\gamma^{\frac{-1}{1-\alpha}}\right)>1, f\left(\gamma^{\frac{1}{\alpha}}\right)>1$.

The first two inequalities in 3 ensures that limited participation equilibrium exists at least for some values of $m$. The following proposition characterizes the conditions on liquidity shocks for the existence of fragility in the market:

Proposition 1. Given 3, the necessary and sufficient conditions in terms of the liquidity shocks $\delta_{0}$ and $\delta_{1}$ that ensure the existence of fragility in the asset market at least for some

\footnotetext{
${ }^{8}$ For example if we have $m<\frac{\delta_{1}}{\delta_{0}}$ or $f(m)<1$, a no-trade equilibrium doesn't exist.

${ }^{9}$ For this reason given the remaining measures of buyers and sellers at $t=1$, equilibrium at $t=1$ is always unique and efficient.
} 


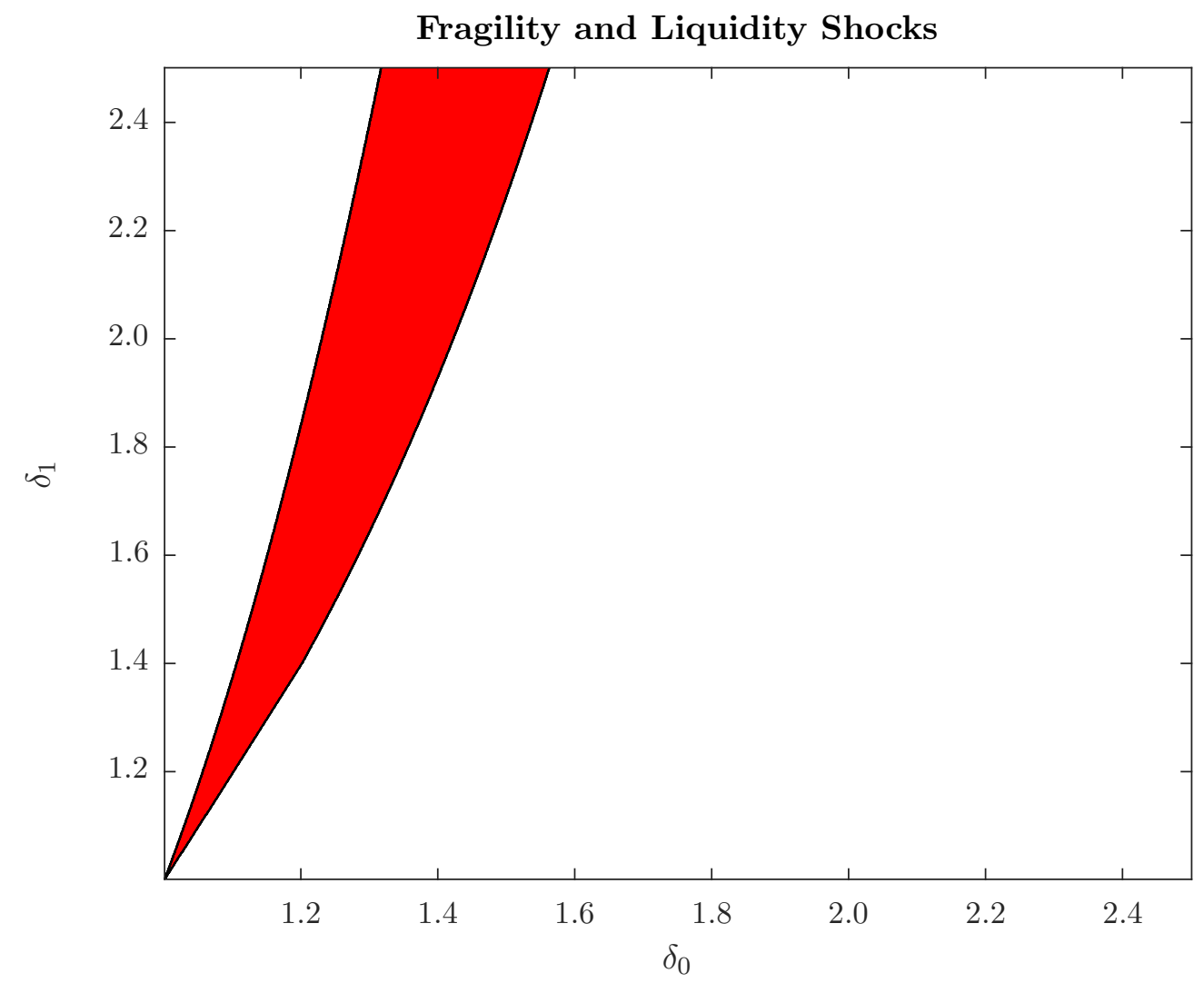

Figure 1: The region with red illustrates the values of liquidity shocks for which markets are fragile for $\alpha=0.55$ and $\gamma=0.3$.

values of $m$ is:

$$
f\left(\frac{\delta_{1}}{\delta_{0}}\right)<1 \Longleftrightarrow \gamma\left(\frac{\delta_{1}}{\delta_{0}}\right)^{-\alpha}<\frac{\delta_{0}-1}{\delta_{1}-1}
$$

The above condition implies:

$$
1<\frac{\delta_{1}}{\delta_{0}}<\gamma^{\frac{-1}{1-\alpha}}
$$

Moreover there is no fragility for $m<1$, if $f(1)>1$.

Proposition 1 states that to have fragility in the market the future liquidity shock should be bigger than the current one. This has to be case in order to raise the value of waiting relative to participation at the initial date. The total expected surplus of waiting can be no 
less than that of participating at time zero, only if sellers don't discount the value of their future participation at $t=1$. Otherwise, sellers and buyers don't find it optimal to delay their trade at $t=0$.

For fixed levels of $m$ and current liquidity shock, $\delta_{0}$, an increasing $\delta_{1}$ changes the profile of equilibria as follows. For low levels of $\delta_{1}$, the only equilibrium is the full participation equilibrium. As $\delta_{1}$ increases further and within the medium range of the future liquidity shock indicated by Proposition 1, both types of full and limited participation equilibria exist and market becomes fragile. Increasing $\delta_{1}$ even further leads to an equilibrium in which there is no trade at $t=0$.

This is similar to other environments such as models of currency attacks where multiple equilibria is an outcome when fundamentals are within a medium range, i.e., not too weak or too strong. It is also important to note that a higher liquidity shock today, makes it less likely to have market fragility. Non-extreme values of $\delta_{1} / \delta_{0}$ are needed for fragility because opportunities to trade in the current and future dates should be relatively comparable. This has to be the case for the externalities of each agent's decision to trade to have any effect on others.

The next result shows how fragility depends on $m$, the ratio of sellers to buyers or the size of (potential) supply relative to demand for the asset. Using Proposition 1, we restrict our attention to the case $f(1)<1$ where markets with $m<1$ can also be fragile:

Proposition 2. Assume that $f(1)<1$. Given a set of all other parameters, $\left\{\gamma, \delta_{0}, \delta_{1}\right\}$, there exist a quadruple $\{\underline{\underline{m}}, \bar{m}, \underline{\underline{m}}, \overline{\bar{m}}\}$ satisfying:

$$
\left\{\begin{array}{l}
\gamma^{\frac{1}{\alpha}}<\underline{m}<\bar{m}<1 \\
1<\underline{\underline{m}}<\overline{\bar{m}}<\gamma^{\frac{-1}{1-\alpha}}
\end{array}\right.
$$

such that markets are fragile if and only if $m$ satisfies one of the two following conditions:

$$
\left\{\begin{array}{l}
m<1, \quad \underline{m}<m<\bar{m} \\
m>1, \quad \underline{\underline{m}}<m<\overline{\bar{m}}
\end{array}\right.
$$

The above proposition suggests that there should be a minimum imbalance between the supply and demand for the asset for the market to be fragile. This is because when $m$ is close to one, participation decisions at $t=0$ by agents don't have any substantial impact on 
the (inverse of) market tightness, $\sigma_{1}^{*}$, at $t=1$ and reservation utility of others at $t=0$ : in the limit when $m=1$, there will be no impact and $\sigma_{1}^{*}=1$ regardless of what happens in the market at $t=0$.

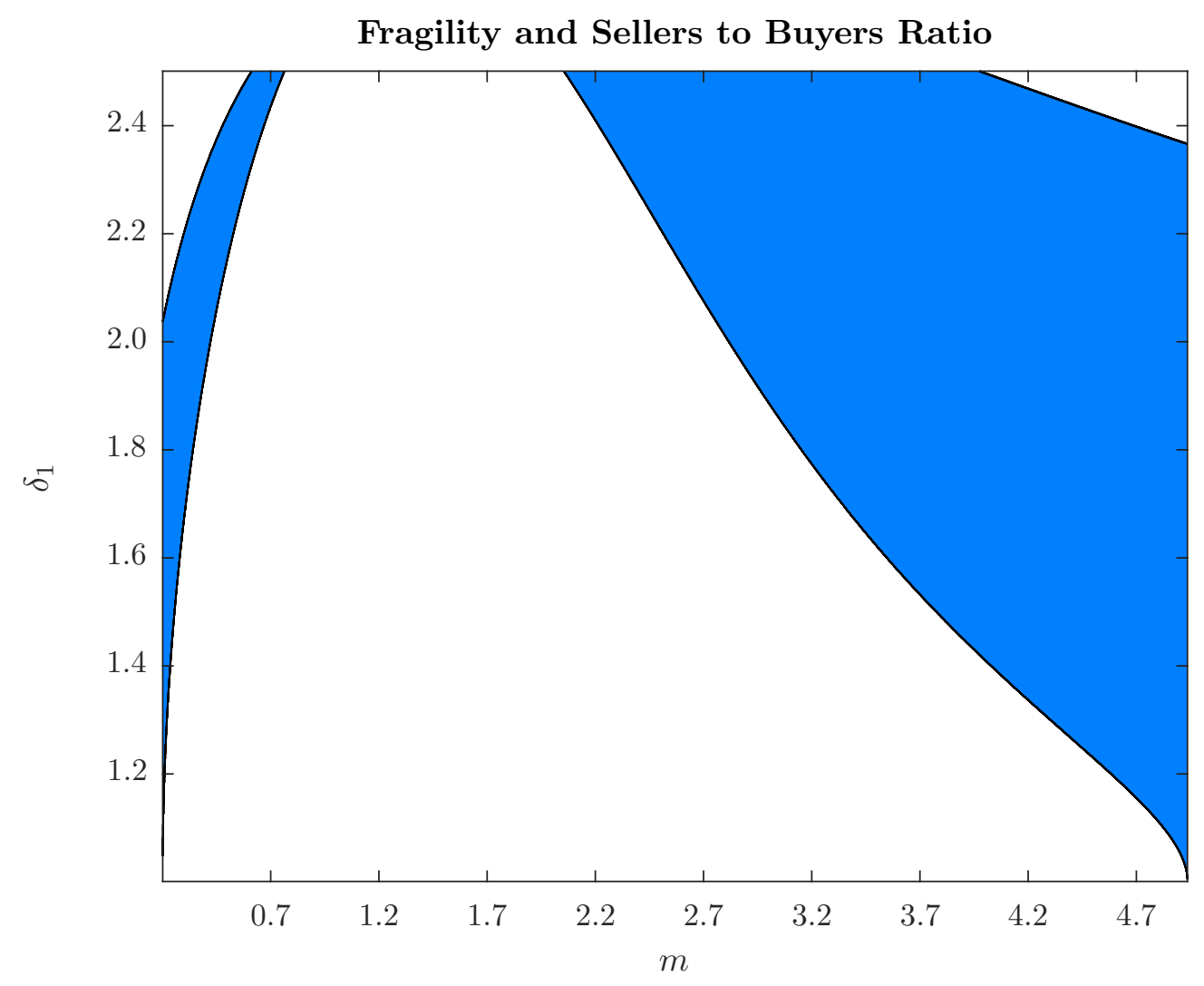

Figure 2: The blue region illustrates the values of $m$ for any level of $\delta_{1}$ for which markets are fragile. Note that $\delta_{0}=1.6, \alpha=0.5$ and $\gamma=0.45$.

Finally, we examine whether more or less liquid markets are prone to fragility. We focus on the case $m<1$, where fire-sale can happen only by switching from a run to a delayed equilibrium. Given $m<1$, the main parameter determining market liquidity in the model is $\gamma$. Higher values of $\gamma$ means higher probability of being matched to a trading partner and hence higher market liquidity (given a certain level of participation). Fixing all other parameters one can derive the following conditions for values of $\gamma$ for which markets are fragile:

LEMma 4. For a given set of parameters $\left\{\alpha, \delta_{0}, \delta_{1}, m\right\}$, the matching efficiency, $\gamma$, has to 
satisfy the following necessary conditions for the existence of multiple equilibria:

$$
\frac{m^{\alpha}\left(\delta_{0}-1\right) \delta_{1}}{\left((1-\alpha) \delta_{1}+\alpha m \delta_{0}\right)\left(\delta_{1}-1\right)} \leq \gamma \leq \frac{\left(\delta_{0}-1\right) \delta_{1}}{\left((1-\alpha) \delta_{1}+\alpha \delta_{0}\right)\left(\delta_{1}-1\right)}
$$

Similar to the degree of imbalance in the market, too liquid or too illiquid markets don't feature multiplicity or fire-sale. For coordination failures and multiplicity, the present externalities need to create complementarity between participation decision of sellers and buyers in the market. This can't happen when liquidity, imbalance in the market or liquidity shocks are too high or too low. To see why assume $m<1$ and consider a situation in which measure $s_{0}$ and $b_{0}$ of sellers and buyers have already decided to participate in the market at $t=0$. Now consider a buyer who is deciding whether to participate in the market at $t=0$. We have $\sigma_{0}=\frac{s_{0}}{b_{0}}$ and that:

$$
\sigma_{1}=\frac{m-\gamma s_{0} \sigma_{0}^{-\alpha}}{1-\gamma s_{0} \sigma_{0}^{-\alpha}}
$$

It is easy to show that the marginal change in $\sigma_{1}$ due to a change in $\sigma_{0}$ as a result of a buyer's participating decision (holding $s_{0}$ fixed) is:

$$
\left.\frac{d \sigma_{1}}{d \sigma_{0}}\right|_{s_{0}}\left(b_{0}\right)=\frac{\alpha \gamma s_{0} \sigma_{0}^{-(1+\alpha)}(1-m)}{\left(1-\gamma s_{0} \sigma_{0}^{-\alpha}\right)^{2}}
$$

Note that $\gamma s_{0} \sigma_{0}^{-\alpha}<1$ since $\gamma \sigma_{0}^{-\alpha}$ is a probability. Finally we can write the relative marginal change in probabilities of meeting a partner for sellers and buyers as follows:

$$
\left\{\begin{array}{l}
\left.\frac{d q_{1}^{S}}{d q_{0}^{S}}\right|_{s_{0}}\left(b_{0}\right)=\left(\frac{\sigma_{1}}{\sigma_{0}}\right)^{-(1+\alpha)} \frac{d \sigma_{1}}{d \sigma_{0}} \\
\left.\frac{d q_{1}^{B}}{d q_{0}^{B}}\right|_{s_{0}}\left(b_{0}\right)=\left(\frac{\sigma_{1}}{\sigma_{0}}\right)^{-\alpha} \frac{d \sigma_{1}}{d \sigma_{0}}
\end{array}\right.
$$

When $m<1$ a marginal decrease in $b_{0}$, as a result of a buyer's decision to wait, implies $d q_{1}^{B}>0$ and $d q_{0}^{B}>0$ while $d q_{1}^{S}<0$ and $d q_{0}^{S}<0$. In order to have complementarity in waiting, these marginal changes should encourage other buyers and sellers to wait as well. 
This is the case if $\frac{d q_{1}^{B}}{d q_{0}^{B}}$ isn't too low and $\frac{d q_{1}^{S}}{d q_{0}^{S}}$ isn't too high, otherwise sellers and buyers would prefer not to wait and to trade at $t=0$. Given the above expressions for the relative changes in probabilities, this suggests that $\frac{d \sigma_{1}}{d \sigma_{0}}$ should not be too high or too low. This derivative is a product of two terms: $1-m$ and $\frac{\alpha \gamma s_{0} \sigma_{0}^{-(1+\alpha)}}{\left(1-\gamma s_{0} \sigma_{0}^{-\alpha}\right)^{2}}$. In order to have the product in a medium range, $1-m$ and $\gamma$ should be in a medium range as well, as the latter is increasing in $\gamma$.

In contrast to s buyer's waiting, when $m<1$, a seller's decision to wait until $t=1$ reduces $\sigma_{0}$ but increases $\sigma_{1}$. This unambiguously encourages other sellers to trade at $t=0$, implying that sellers' decisions to wait are substitute rather than complement.

It's important to note that any change in $b_{0}$ and consequently $\sigma_{0}$ and $\sigma_{1}$ not only affects probabilities but also $p_{0}$ which we have ignored in the above argument for simplicity. When $m<1$, a buyer's decision to wait at $t=0$ leads to higher reservation utility for other buyers. By 19, we know that this results in a lower $p_{0}$ while $p_{1}$ is independent of agents' actions at $t=0$. As long as the decrease in the price at time zero is not too big there will be complementarity among buyers' waiting decisions. The size of the decline in time zero price depends on $\frac{d \sigma_{1}}{d \sigma_{0}}$ given above. Again, this implies that medium values of $m$ and $\gamma$ are needed to ensure that both buyers and sellers will find it optimal to postpone their trade.

\subsubsection{Price Impact of Fire-Sale}

An important aspect of fire-sale is the size of its price impact. Sizable price impact may be both a symptom and a cause of deteriorating conditions in financial markets. For example, highly depressed asset prices lead to significant balance sheet distress for some banks during the recent crisis. This in turn hindered socially beneficial activities such as lending, market making and liquidity provision for other financial entities.

In this subsection, I characterize how $m$ and $\gamma$, parameters governing liquidity and market tightness, as well as the level of fundamentals $\delta_{0}$ and $\delta_{1}$ affect the size of the price impact in fire-sale. For expositional clarity, below I define what I mean by price impact of fire-sales.

DEFINITION 4. When markets are fragile, the price impact of fire-sale is defined as the percentage decline in the asset price when agents switch from the high asset price to the firesale equilibrium. More precisely, the price impact is defined as $\Delta_{p_{0}} \equiv \frac{\left|p_{0}^{* D}-p_{0}^{* R}\right|}{p_{0}^{* D}}$ when fire-sale happens in a $\underline{\text { run }}$, and as $\Delta_{p_{0}} \equiv \frac{\left|p_{0}^{* D}-p_{0}^{* R}\right|}{p_{0}^{* R}}$ when it happens in a delayed equilibrium.

The following proposition shows how liquidity and the ratio of sellers to buyers affect the size of the price impact.

Proposition 3. Assume that markets are fragile for a set of parameters, $\left\{m, \gamma, \delta_{0}, \delta_{1}, \alpha\right\}$. 


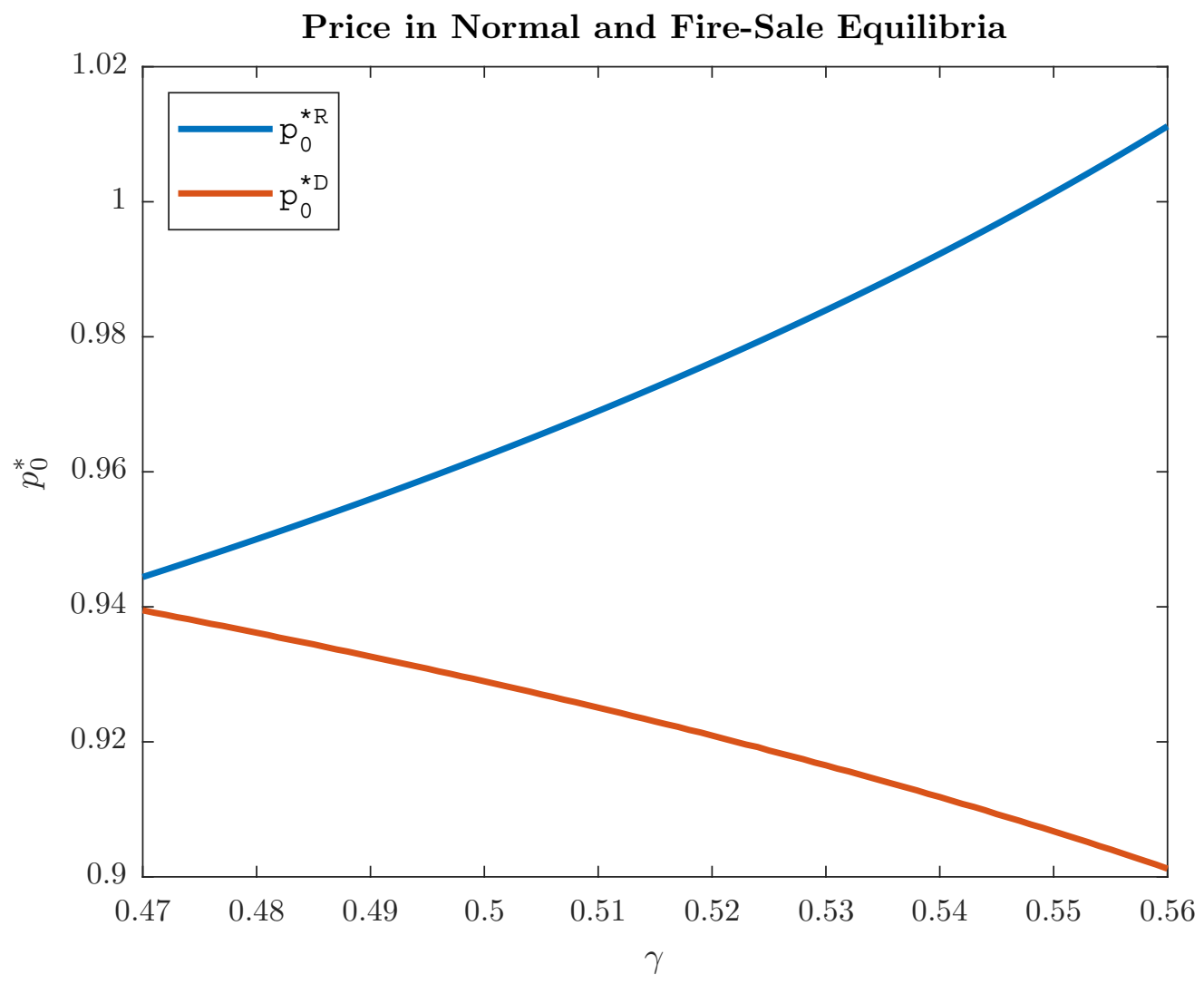

Figure 3: $p_{0}^{* D}$ and $p_{0}^{* R}$ for different values of $\gamma$. Other parameter values are $\alpha=0.5, \delta_{0}=1.55$, $\delta_{1}=2$ and $m=0.5$.

Then $\frac{d \Delta_{p_{0}}}{d m}>0$ if fire-sale is a run (if $m>1$ ) and $\frac{d \Delta_{p_{0}}}{d m}<0$ if fire-sale is a delayed equilibrium (if $m<1)$. This result implies:

$$
\frac{d \Delta_{p_{0}}}{d|m-1|}>0
$$

The effect of $\gamma$ is as follows. When fire-sale happens in a delayed equilibrium (if $m<1$ ), we have $\frac{d \Delta_{p_{0}}}{d \gamma}>0$. When fire-sale happens in a $\underline{\text { run }}$ equilibrium, if $m>\frac{\delta_{1}}{\delta_{0}}$, a sufficient condition to have $\frac{d \Delta_{p_{0}}}{d \gamma}>0$ is:

$$
\left(\frac{\left(m-\gamma m^{1-\alpha}\right)\left(1-\gamma m^{1-\alpha}\right)}{\gamma m^{1-\alpha}(m-1)}-\alpha\right)\left(\frac{1}{\delta_{0}}-\frac{m-\gamma m^{1-\alpha}}{\left(1-\gamma m^{1-\alpha}\right) \delta_{1}}\right)<1
$$

The price impact of fire-sale, $\Delta_{p_{0}}$, is unambiguously higher when there is more imbalance between the buy and sell sides, i.e., higher $|m-1|$, in the market. We know by 17 that $p_{0}^{* D}$ doesn't depend on $m$. Hence what drives the changes in $\Delta_{p_{0}}$ is what happens to $p_{0}^{* R}$. Now 
without any loss of generality, consider the case where $m>1$. Higher $m$ in this case implies a lower market tightness at $t=1$ and in turn a lower reservation utility at $t=0$ for sellers. This suggests that higher $m$ should depress the price in the run equilibrium.

COROLlary 1. Let $\nu_{0}^{* D}$ and $\nu_{0}^{* R}$ denote the volume of trade at $t=0$ for delayed and $\underline{\text { run }}$ equilibria respectively. When $m<1$ and market is fragile, $\nu_{0}^{* D}$ is decreasing and $\nu_{0}^{* R}$ is increasing in $\gamma$. This also implies that the impact on trade volume $\frac{\nu_{0}^{* R}-\nu_{0}^{* D}}{\nu_{0}^{* R}}$ is increasing in $\gamma$.

The above corollary states that when sellers are the short side of the market, more liquid markets experience higher prices and trade volume in normal times and lower prices and trade volume during fire-sales. Some recent works such as Boudoukh et al. (2016) and Gorton (2010) document a puzzling reversal of the spread between more and less liquid securities during crisis time. If these reversals are accompanied by lower trade volume, these observations would be consistent with the predictions above.

\subsection{Welfare Analysis}

In this section, we look at the welfare properties of different types of equilibria when markets are fragile. To this end and as the preferences are linear, we can assume a planner who aims at maximizing the aggregate sum of the trade surplus in units of time zero consumption goods. This amounts to summing up the time zero consumption equivalent of utilities of all agents:

$$
W \equiv \bar{U}_{0}^{B}+\frac{m}{\delta_{0}} \bar{U}_{0}^{S}
$$

The planner can make lump sum taxes and transfers at $t=0$ to improve sellers and buyers welfare. The following lemma shows how we can rank equilibria according to the welfare measure defined in 25:

LEMma 5. If $W_{i}$ and $W_{j}$ are total welfare for two different equilibria $i$ and $j$ where $W_{j}>W_{i}$, the planner can design transfers in $j$ to achieve an allocation $j^{\prime}$ that is Pareto superior to $i$.

I now derive the total welfare in the case of a run equilibrium. Using the objective function 5 and FOC for a run equilibrium in 12 we have: 


$$
\left\{\begin{array}{l}
\bar{U}_{0}^{B}=\gamma \sigma_{0}^{* 1-\alpha}\left(d_{2}-\frac{1}{\delta_{0}} \bar{U}_{1}^{S}-\bar{U}_{1}^{B}\right)-(1-\alpha) \gamma \sigma_{0}^{* 1-\alpha}\left(d_{2}-\frac{1}{\delta_{0}} \bar{U}_{1}^{S}-\bar{U}_{1}^{B}\right)+\bar{U}_{1}^{B}, \\
\frac{1}{\delta_{0}} \bar{U}_{0}^{S}=(1-\alpha) \gamma \sigma_{0}^{*-\alpha}\left(d_{2}-\frac{1}{\delta_{0}} \bar{U}_{1}^{S}-\bar{U}_{1}^{B}\right)+\frac{1}{\delta_{0}} \bar{U}_{1}^{S}
\end{array}\right.
$$

Using the fact that there is full participation in a run equilibrium, i.e., $\sigma_{0}^{*}=m$, we can compute the welfare by adding the two terms in 26 and simplifying as follows:

$$
W=\left(\bar{U}_{1}^{B}+\frac{\bar{U}_{1}^{S}}{\delta_{0}} m\right)+\gamma m^{1-\alpha}\left(d_{2}-\frac{1}{\delta_{0}} \bar{U}_{1}^{S}-\bar{U}_{1}^{B}\right)
$$

The first term in 27 is the sum of (time zero consumption equivalent) reservation utilities of both sellers and buyers. The second term is the product of total number of transactions at $t=0$ which is $\gamma m^{1-\alpha}$ and the total extra trade surplus (net of reservation utilities) of a match between a seller and a buyer.

Note that when there is multiplicity, 27 can be applied to both run and delayed equilibria. In the case of a delayed equilibrium, the extra surplus $d_{2}-\frac{1}{\delta_{0}} \bar{U}_{1}^{S}-\bar{U}_{1}^{B}$ is equal to zero and total welfare is equal to the sum of reservation utilities. Therefore, and based on Lemma 5 , we can use this measure to rank different equilibria when there is fragility. The following proposition establishes the main result of this section:

Proposition 4. Given a set of parameters $\left\{\gamma, \delta_{0}, \delta_{1}\right\}$ for which market is fragile, the following are true for the run and delayed equilibria. When $m>1$ :

$$
m>1 \Rightarrow W^{D}\left(m, \gamma, \delta_{0}, \delta_{1}\right)>W^{R}\left(m, \gamma, \delta_{0}, \delta_{1}\right)
$$

And there exists $0<\xi<1$ such that for $m<1$ :

$$
\xi \leq m<1 \Rightarrow W^{D}\left(m, \gamma, \delta_{0}, \delta_{1}\right)<W^{R}\left(m, \gamma, \delta_{0}, \delta_{1}\right)
$$

Where $W^{R}$ and $W^{D}$ denote the total welfare for the run and delayed equilibria respectively.

Lemma 5 and Proposition 4 imply that the run and delayed equilibria can be ranked according to welfare in a meaningful sense. We note that when market is fragile, the equilibrium with the fire-sale price is worse in terms of welfare as long as sellers to buyers ratio is not too low: 
COROLlary 2. When market is fragile, the equilibrium with fire-sale has the lower total welfare for all $m>\xi$ where $\xi<1$ is defined above.

\section{Policy}

Different types of policies may be used to eliminate the possibility of fire-sale equilibria in this model. For example, when market is fragile, a policy to purchase the asset at a minimum price $p_{0}^{\min }$ can always implement the equilibrium with the higher asset price. This policy works by eliminating the possibility of fire-sale as long as $p_{0}^{L}<p_{0}^{\min }<p_{0}^{H}$, where $p_{0}^{H}$ and $p_{0}^{L}$ are time zero price of asset in the two equilibria with high and low asset prices. A credible commitment by the government or the central bank is enough to eliminate the fir-sale and no asset purchase needs to take place in equilibrium. Asset purchasing policies implemented after the financial crisis as part of different quantitative easing (QE) programs are relevant examples in this regard.

Policies that change the market structure or trading platforms can affect market fragility and the possibility of fire-sale as well. Standardization of the securities traded in the market, for instance, can increase liquidity by affecting the efficiency of matching (higher $\gamma$ ), and by broadening the buyer base of the asset (higher $m$ ). If the impact on liquidity is strong enough, this can eliminate the fire-sale equilibrium. Nonetheless, if the impact is modest and market liquidity is initial very low, an increase in liquidity may end up making the market fragile and subject to fire-sale (proposition Proposition 4). Higher transparency in OTC markets as well as introduction of centralized clearing platforms may have similar ambiguous effects on market liquidity and hence on fragility. ${ }^{10}$

\section{Conclusion}

This paper shows that decentralized asset markets can be fragile and prone to fire-sales when they are subjected to liquidity shocks that are increasing over time. I demonstrate conditions under which markets are fragile: liquidity shocks that are increasing in magnitude over time, a medium degree of liquidity as well as imbalance between sellers and buyers in the market. These fire-sales may be inefficient in that they lead to a suboptimally low aggregate trade surplus relative to the equilibrium with the higher asset price.

I have abstracted from balance sheet effects of fire-sales in this model. One can extend the model to make fire-sales worsen the balance sheets of the sellers. While the key insights

\footnotetext{
${ }^{10}$ See Loon and Zhong (2016) for evidence on the effects of Dodd-Frank on OTC transaction liquidity.
} 
of the model should hold in such an extended version, these balance sheet externalities can deepen the fire-sale discount and exacerbate the negative welfare effects. It may be also worth to examine whether the balance sheet effects can widen the range of fundamentals for which markets are fragile.

Another extension which might be of interest is where agents hold a portfolio of assets with different liquidity. In the real world, sellers and buyers typically hold a portfolio of assets with different risk, return and liquidity. On the one hand, holding highly liquid assets, e.g., treasuries, can be a buffer against liquidity shocks as their price won't be subject to fire-sales. But they may also crowd out buyers and reduce the effective demand for the less liquid assets in the market. This concern can be even more important if agents are risk averse and more liquid assets are also safer. Whether and under what conditions holding more liquid assets can make markets less fragile is a question for future research. 


\section{References}

Bernardo, A. E. and I. Welch (2004, February). Liquidity and financial market runs. Quarterly Journal of Economics 119(1), 135-158.

Boudoukh, J., J. Brooks, M. Richardson, and Z. Xu (2016, August). The complexity of liquidity: The extraordinary case of sovereign bonds.

Chang, B. (2018, January). Adverse selection and liquidity distortion. The Review of Economic Studies 85(1), 275-306.

Chernenko, S., S. G. Hanson, and A. Sunderam (2014, December). The rise and fall of demand for securitization.

Diamond, D. W. and R. Rajan (2011, May). Fear of fire sales, iliiquidity seeking, and credit freezes. The Quarterly Journal of Economics 126(2), 557-591.

Dow, J. and J. Han (2018, February). The paradox of financial fire sales: The role of arbitrage capital in determining liquidity. Journal of Finance 73(1), 229-274.

Duffie, D. (2010, August). Presidential address: Asset price dynamics with slow-moving capital. The Journal of Finance 65(4), 1237-1267.

Gorton, G. (2010, February). Questions and answers about the financial crisis. Prepared for the U.S. Financial Crisis Inquiry Commission.

Guerrieri, V. (2010). Inefficient unemployment dynamics under asymmetric information. IMF Economic Review 58, 118-156.

Guerrieri, V. and R. Shimer (2014, July). Dynamic adverse selection: A theory of illiquidity, fire sales, and flight to quality. American Economic Review 104 (7), 1875-1908.

Hanson, S. G. and A. Sunderam (2013, June). Are there too many safe securities? securitization and the incentives for information production. The Quarterly Journal of Economics 108(3), 556-584.

He, Z., I. G. Khang, and A. Krishnamurthy (2010). Balance sheet adjustments during the 2008 crisis. IMF Economic Review 58, 118-156.

Loon, Y. C. and Z. Zhong (2016, March). Does dodd-frank affect otc transaction costs and liquidity? evidence from real-time cds trade reports. Journal of Financial Econoics 119(3), 645-672. 
Obstfeld, M. (1996). Models of currency crises with self-fulfilling features. European Economic Review 40, 1037-1047.

Park, S. Y. (2011). The size of the subprime shock.

Shleifer, A. and R. W. Vishny (1992, September). Liquidation values and debt capacity: A market equilibrium approach. Journal of Finance 47(4), 1343-1366.

Shleifer, A. and R. W. Vishny (1997, March). The limits of arbitrage. Journal of Finance 52(1), 35-55.

Vayanos, D. and P.-O. Weill (2008, June). A search-based theory of the on-the-run phenomenon. The Journal of Finance 63(3), 1361-1398. 


\section{A Appendix: Proofs}

Proof of Lemma 1. For the last part of the proposition, note that we have $\nu_{0}^{R}>\nu_{0}^{D}$, which in the case of $m<1$ implies:

$$
\sigma_{1}^{* R}=\frac{m-\nu_{0}^{R}}{1-\nu_{0}^{R}}<\frac{m-\nu_{0}^{D}}{1-\nu_{0}^{D}}=\sigma_{1}^{* D}
$$

Given the derivations in the text, this implies that $p_{0}^{* R}>p_{0}^{* D}$. The rest of the proposition is proven in the text.

Proof of Lemma 2. We solve the equilibrium backward. Suppose that $1-s$ and $m-s$ are measures of buyers and sellers at $t=1$ who haven't already traded in the market, where $s$ is the volume of trade at $t=0$.

Consider the case of $m>1$ first. Let $p_{1}$ denote the price in the market at $t=1$. The demand curve for asset is:

$$
\left\{\begin{array}{l}
0 \leq p_{1}<d_{2} \Rightarrow a_{1}^{d}\left(p_{1}\right)=1-s \\
p_{1}=d_{2} \Rightarrow a_{1}^{d}\left(p_{1}\right) \in[0,1-s] \\
p_{1}>d_{2} \Rightarrow a_{1}^{d}\left(p_{1}\right)=0
\end{array}\right.
$$

Similarly supply curve at $t=1$ is:

$$
\left\{\begin{array}{l}
0 \leq p_{1}<\frac{d_{2}}{\delta_{1}} \Rightarrow a_{1}^{s}\left(p_{1}\right)=0 \\
p_{1}=\frac{d_{2}}{\delta_{1}} \Rightarrow a_{1}^{s}\left(p_{1}\right) \in[0, m-s] \\
p_{1}>\frac{d_{2}}{\delta_{1}} \Rightarrow a_{1}^{s}\left(p_{1}\right)=m-s
\end{array}\right.
$$

Where $a^{d}$ and $a^{s}$ are demand and supply for the asset. It is easy to check that the only equilibrium price at $t=1$ where supply and demand for the asset are equal is $p_{1}=\frac{d_{2}}{\delta_{1}}$. And in equilibrium all $1-s$ buyers participate in the market while only $1-s$ measure of sellers trade and the rest $m-1$ stay out (and they will be indifferent between the two options). 
Moving to $t=0$, the demand supply are:

$$
\left\{\begin{array}{l}
0 \leq p_{0}<\frac{d_{2}}{\delta_{1}} \Rightarrow a_{0}^{d}\left(p_{0}\right)=1 \\
p_{0}=\frac{d_{2}}{\delta_{1}} \Rightarrow a_{0}^{d}\left(p_{0}\right) \in[0,1] \\
p_{0}>\frac{d_{2}}{\delta_{1}} \Rightarrow a_{0}^{d}\left(p_{0}\right)=0
\end{array}\right.
$$

Similarly supply curve at $t=0$ is:

$$
\left\{\begin{array}{l}
0 \leq p_{0}<\frac{d_{2}}{\delta_{0}} \Rightarrow a_{0}^{s}\left(p_{0}\right)=0 \\
p_{0}=\frac{d_{2}}{\delta_{0}} \Rightarrow a_{0}^{s}\left(p_{0}\right) \in[0, m] \\
p_{0}>\frac{d_{2}}{\delta_{0}} \Rightarrow a_{0}^{s}\left(p_{0}\right)=m
\end{array}\right.
$$

It is easy to see that equilibrium price and quantities are as follows:

$$
\left\{\begin{array}{l}
\delta_{0}>\delta_{1} \Rightarrow p_{0}=\frac{d_{2}}{\delta_{0}}, \quad a_{0}^{s}=a_{0}^{d}=1 \\
\delta_{0}=\delta_{1} \Rightarrow p_{0}=\frac{d_{2}}{\delta_{0}}, \quad a_{0}^{s}=a_{0}^{d} \in[0,1] \\
\delta_{0}<\delta_{1} \Rightarrow p_{0} \in\left[\frac{d_{2}}{\delta_{1}}, \frac{d_{2}}{\delta_{0}}\right], \quad a_{0}^{s}=a_{0}^{d}=0
\end{array}\right.
$$

Therefore whenever there is any trade at $t=0$, the equilibrium price is unique and equal to $\frac{d_{2}}{\delta_{0}}$.

Now consider the case of $m<1$. Demand and supply curves at $t=1$ are defined as before. But because $m<1$, the unique price will be $p_{1}=d_{2}$. At $t=0$ we have:

$$
\left\{\begin{array}{l}
0 \leq p_{0}<d_{2} \Rightarrow a_{0}^{d}\left(p_{0}\right)=1 \\
p_{0}=d_{2} \Rightarrow a_{0}^{d}\left(p_{0}\right) \in[0,1] \\
p_{0}>d_{2} \Rightarrow a_{0}^{d}\left(p_{0}\right)=0
\end{array}\right.
$$


Similarly supply curve at $t=0$ is:

$$
\left\{\begin{array}{l}
0 \leq p_{0}<\frac{\delta_{1} d_{2}}{\delta_{0}} \Rightarrow a_{0}^{s}\left(p_{0}\right)=0 \\
p_{0}=\frac{\delta_{1} d_{2}}{\delta_{0}} \Rightarrow a_{0}^{s}\left(p_{0}\right) \in[0, m] \\
p_{0}>\frac{\delta_{1} d_{2}}{\delta_{0}} \Rightarrow a_{0}^{s}\left(p_{0}\right)=m
\end{array}\right.
$$

And therefore the equilibrium in this case will be:

$$
\left\{\begin{array}{l}
\delta_{0}>\delta_{1} \Rightarrow p_{0}=d_{2}, \quad a_{0}^{s}=a_{0}^{d}=m \\
\delta_{0}=\delta_{1} \Rightarrow p_{0}=d_{2}, \quad a_{0}^{s}=a_{0}^{d} \in[0, m] \\
\delta_{0}<\delta_{1} \Rightarrow p_{0} \in\left[d_{2}, \frac{\delta_{1} d_{2}}{\delta_{0}}\right], \quad a_{0}^{s}=a_{0}^{d}=0
\end{array}\right.
$$

And again whenever there is trade at $t=0$, the equilibrium price is unique which completes the proof.

Proof of Lemma 3. Given 11, if $d_{2}-\frac{1}{\delta_{0}} \bar{U}_{1}^{S}-\bar{U}_{0}^{B}<0$ then $\sigma_{0}^{*}=0$ is optimal for buyers which implies no trade at $t=0$. Hence we focus on the case $d_{2}-\frac{1}{\delta_{0}} \bar{U}_{1}^{S}-\bar{U}_{0}^{B} \geq 0$.

Consider the case of $d_{2}-\frac{1}{\delta_{0}} \bar{U}_{1}^{S}-\bar{U}_{0}^{B}>0$ first. In this case, if $\bar{U}_{0}^{S}-\bar{U}_{1}^{S}=0$ buyers will set $q_{0}^{B}\left(\sigma_{0}^{*}\right)=1$. This needs either $m \leq \gamma^{\frac{1}{\alpha}}$ or $m \geq \gamma^{\frac{-1}{1-\alpha}}$ which cannot be the case given Assumption 2. To see this note that $m>\frac{m-\gamma m^{1-\alpha}}{1-\gamma m^{1-\alpha}}$ for $m<1$ and $m<\frac{m-\gamma m^{1-\alpha}}{1-\gamma m^{1-\alpha}}$ for $m>1$. Therefore, we need to have $\bar{U}_{0}^{S}-\bar{U}_{1}^{S}>0$. This implies the FOC in 12 are the optimality conditions for buyers. Solving for $\bar{U}_{0}^{B}$ and $\bar{U}_{0}^{S}$ gives:

$$
\left\{\begin{array}{l}
\bar{U}_{0}^{B}=\alpha \gamma \sigma_{0}^{* 1-\alpha}\left(d_{2}-\frac{1}{\delta_{0}} \bar{U}_{1}^{S}-\bar{U}_{0}^{B}\right)+\bar{U}_{1}^{B} \\
\frac{\bar{U}_{0}^{S}}{\delta_{0}}=(1-\alpha) \gamma \sigma_{0}^{*-\alpha}\left(d_{2}-\frac{1}{\delta_{0}} \bar{U}_{1}^{S}-\bar{U}_{0}^{B}\right)+\frac{\bar{U}_{1}^{S}}{\delta_{0}}
\end{array}\right.
$$

This shows that $V_{0}^{B}=\bar{U}_{0}^{B}>\bar{U}_{1}^{B}$ and $V_{0}^{S}=\bar{U}_{0}^{S}>\bar{U}_{1}^{S}$ and that there is full participation by buyers and sellers in equilibrium. Hence in equilibrium we have $\sigma_{0}^{*}=m$ which also implies 
$\sigma_{1}^{*}=\frac{m-\gamma m^{1-\alpha}}{1-\gamma m^{1-\alpha}}$ and:

$$
\left\{\begin{array}{l}
\mu_{0}^{* S}=m, \quad \mu_{0}^{* B}=1, \\
\mu_{1}^{* S}=m-\gamma m^{1-\alpha}, \quad \mu_{1}^{* B}=1-\gamma m^{1-\alpha}
\end{array}\right.
$$

Now consider the case $d_{2}-\frac{1}{\delta_{0}} \bar{U}_{1}^{S}-\bar{U}_{0}^{B}=0$. We must have $\bar{U}_{0}^{S}-\bar{U}_{1}^{S}=0$, otherwise buyers will not enter the market and there will be no trade at $t=0$. Therefore, in this case both buyers and sellers are indifferent between participating in or staying out of the market at time zero. If there exists a solution to $d_{2}-\frac{1}{\delta_{0}} \bar{U}_{1}^{S}-\bar{U}_{0}^{B}=0$ in terms of $\sigma_{1}^{*}$ such that $\sigma_{1}^{*} \in\left[\frac{m-\gamma m^{1-\alpha}}{1-\gamma m^{1-\alpha}}, m\right]$ when $m<1$ or $\sigma_{1}^{*} \in\left[m, \frac{m-\gamma m^{1-\alpha}}{1-\gamma m^{1-\alpha}}\right]$ when $m>1$, we will have the following:

$$
\sigma_{1}^{*}=\frac{m-\gamma \mu_{0}^{* S^{1-\alpha}} \mu_{0}^{* B^{\alpha}}}{1-\gamma \mu_{0}^{* S^{1-\alpha}} \mu_{0}^{* B^{\alpha}}} \Rightarrow \gamma \mu_{0}^{* S^{1-\alpha}} \mu_{0}^{* B^{\alpha}}=\frac{m-\sigma_{1}^{*}}{1-\sigma_{1}^{*}}
$$

Which completes the proof.

Proof of Proposition 1. Taking the derivative of $f\left(\sigma_{1}\right)$ gives:

$$
f^{\prime}\left(\sigma_{1}\right)=\frac{\alpha(1-\alpha)\left(\delta_{1}-1\right)}{\delta_{1}} \gamma \sigma_{1}^{-(1+\alpha)}\left\{\sigma_{1}-\frac{\delta_{1}}{\delta_{0}}\right\}
$$

We note that $f\left(\sigma_{1}\right)$ is strictly decreasing for $\sigma_{1}<\frac{\delta_{1}}{\delta_{0}}$ and strictly increasing for $\frac{\delta_{1}}{\delta_{0}}<\sigma_{1}$ and has a minimum at $\frac{\delta_{1}}{\delta_{0}}$. By Assumption 3, we know that $f$ takes values higher than one at the boundaries. Hence the necessary and sufficient condition for the existence of a root is $f\left(\frac{\delta_{1}}{\delta_{0}}\right)<1$. This implies:

$$
\left\{\begin{array}{l}
f\left(\frac{\delta_{1}}{\delta_{0}}\right)=\frac{1}{\delta_{0}}+\gamma\left(\frac{\delta_{1}}{\delta_{0}}\right)^{-\alpha} \frac{\delta_{1}-1}{\delta_{0}}<1 \Longleftrightarrow \\
\gamma\left(\frac{\delta_{1}}{\delta_{0}}\right)^{-\alpha}<\frac{\delta_{0}-1}{\delta_{1}-1}
\end{array}\right.
$$


It is easy to verify that Assumption 3 and Assumption 2 imply $\delta_{1}>\delta_{0}$. This and the above conditions give:

$$
\gamma\left(\frac{\delta_{1}}{\delta_{0}}\right)^{-\alpha}<\frac{\delta_{0}-1}{\delta_{1}-1}<\frac{\delta_{0}}{\delta_{1}} \Rightarrow \frac{\delta_{1}}{\delta_{0}}<\gamma^{\frac{-1}{1-\alpha}}
$$

Therefore $1<\frac{\delta_{1}}{\delta_{0}}<\gamma^{\frac{-1}{1-\alpha}}$. Finally, if $m<1$ we have $\gamma^{\frac{1}{\alpha}}<\frac{m-\gamma m^{1-\alpha}}{1-\gamma m^{1-\alpha}}<m<1$ (Assumption 2). If $f(1)>1$, all values of $f$ in $\left[\gamma^{\frac{1}{\alpha}}, 1\right]$ are strictly higher than one and hence there's no $\sigma_{1} \in\left[\frac{m-\gamma m^{1-\alpha}}{1-\gamma m^{1-\alpha}}, m\right]$ for which $f\left(\sigma_{1}\right)=1$. This completes the proof.

Proof of Proposition 2. Let's define the function $\zeta(m) \equiv \frac{m-\gamma m^{1-\alpha}}{1-\gamma m^{1-\alpha}}$. Taking the derivative we have:

$$
\zeta^{\prime}(m)=\left\{1-\left(\alpha \gamma m^{1-\alpha}+(1-\alpha) \gamma m^{-\alpha}\right)\right\} \frac{1}{\left(1-\gamma m^{1-\alpha}\right)^{2}}>0
$$

The inequality above holds because $\gamma m^{1-\alpha}$ and $\gamma m^{-\alpha}$ are always less than one by Assumption 2. Moreover we have $\zeta(1)=1$ and $\zeta(0)=0$. Hence $\zeta:[0,1] \rightarrow[0,1]$ is an isomorphism and has a well defined inverse on $[0,1]$. Moreover, $\zeta:\left[1, \gamma^{\frac{-1}{1-\alpha}}\right) \rightarrow[1, \infty)$ is also an isomorphism strictly increasing for $1<m<\gamma^{\frac{-1}{1-\alpha}}$.

Consider the case of $m<1$ first. And let $\tilde{\sigma}_{1}$ be the solution to 15 which amounts to $f\left(\tilde{\sigma}_{1}\right)=1$. We know that $\gamma^{\frac{1}{\alpha}}<\tilde{\sigma}_{1}<1$ exists because $f(1)<1$ and $f\left(\gamma^{\frac{1}{\alpha}}\right)>1$ by Assumption 3. We know from the proof of Proposition 1 that $f$ is strictly decreasing for $m<1$. Also we must have that $\tilde{\sigma}_{1} \in[\zeta(m), m]$ (note that $\zeta(m)<m$ for $m<1$ ) which implies:

$$
f(m)<f\left(\tilde{\sigma}_{1}\right)<f(\zeta(m)) \Rightarrow \zeta(m)<\tilde{\sigma}_{1}<m \Rightarrow \tilde{\sigma}_{1}<m<\zeta^{-1}\left(\tilde{\sigma}_{1}\right)
$$

Given that $f\left(\tilde{\sigma}_{1}\right)=1$ and that $\zeta$ is an isomorphism which also imply that $\zeta^{-1}\left(\tilde{\sigma}_{1}\right)<1$.

Next consider the case when $m>1$. Let $\tilde{\tilde{\sigma}}_{1} \in\left[\frac{\delta_{1}}{\delta_{0}}, \gamma^{\frac{-1}{1-\alpha}}\right]$ be the solution to $f\left(\tilde{\tilde{\sigma}}_{1}\right)=1$. We know that such solution exists because of Assumption 3, that $f\left(\frac{\delta_{1}}{\delta_{0}}\right)<f(1)=1$ since $f$ is minimized at $\frac{\delta_{1}}{\delta_{0}}$ and that $f$ is strictly increasing for $\left[\frac{\delta_{1}}{\delta_{0}}, \gamma^{\frac{-1}{1-\alpha}}\right]$ (Proposition 1 ). We must also have $\tilde{\tilde{\sigma}}_{1} \in[m, z e t a(m)]$ which implies $\frac{\delta_{1}}{\delta_{0}}<\zeta(m)$. This gives:

$$
f\left(\tilde{\tilde{\sigma}}_{1}\right)<f(\zeta(m)) \Rightarrow \tilde{\tilde{\sigma}}_{1}<\zeta(m) \Rightarrow \zeta^{-1}\left(\tilde{\tilde{\sigma}}_{1}\right)<m<\zeta^{-1}\left(\gamma^{\frac{-1}{1-\alpha}}\right)<\gamma^{\frac{-1}{1-\alpha}}
$$

The last inequalities are the results of Assumption 2 and the fact that $m<\zeta(m)$ for $m>1$. $\tilde{\tilde{\sigma}}_{1}>\frac{\delta_{1}}{\delta_{0}}>1$ and hence $1<\zeta^{-1}\left(\tilde{\tilde{\sigma}}_{1}\right)$ which completes the proof. 
Proof of Lemma 4. $m<1$ implies that at time $t=1$ inverse of market tightness $\sigma_{1}$ satisfies $m \leq \sigma_{1} \leq 1$. Using the proof of Proposition 1, we know that function $f\left(\sigma_{1}\right)$ is strictly decreasing for $\sigma_{1} \in[m, 1]$. This implies that $f\left(\sigma_{1}\right)=1$ has a solution within $\sigma_{1} \in[m, 1]$ if and only if $f(1) \leq 1$ and $f(m) \geq 1$. These last two conditions can be written as:

$$
\left\{\begin{array}{l}
\frac{1}{\delta_{0}}+\left\{\frac{(1-\alpha)\left(\delta_{1}-1\right)}{\delta_{0}}+\frac{\alpha\left(\delta_{1}-1\right)}{\delta_{1}}\right\} \gamma \leq 1 \\
\frac{1}{\delta_{0}}+\left\{\frac{(1-\alpha)\left(\delta_{1}-1\right)}{\delta_{0}} m^{-\alpha}+\frac{\alpha\left(\delta_{1}-1\right)}{\delta_{1}} m^{1-\alpha}\right\} \gamma \geq 1
\end{array}\right.
$$

Taking $\gamma$ to one side in above and simplifying yields:

$$
\frac{m^{\alpha}\left(\delta_{0}-1\right) \delta_{1}}{\left((1-\alpha) \delta_{1}+\alpha m \delta_{0}\right)\left(\delta_{1}-1\right)} \leq \gamma \leq \frac{\left(\delta_{0}-1\right) \delta_{1}}{\left((1-\alpha) \delta_{1}+\alpha \delta_{0}\right)\left(\delta_{1}-1\right)}
$$

Proof of Proposition 3. First we prove the result for $m$. First, note that $p_{0}^{* D}$ which is a solution to 16doesn't depend on $m$. This implies that the direction of change in $\Delta_{p_{0}}$ depends solely on how $m$ affects $p_{0}^{* R}$. When $m>1$ (fire-sale in a $r u n$ ) we have $\Delta_{p_{0}}=\frac{\left|p_{0}^{* D}-p_{0}^{* R}\right|}{p_{0}^{* D}}$ by definition. We know that:

$$
\sigma_{1}^{* R}=\zeta(m)
$$

where $\zeta(m)$ is defined in the proof of Proposition 2 and is shown to be increasing in $m$. Hence we have $\frac{d \sigma_{1}^{* R}}{d m}>0$. But we also know by 20 that $\frac{d p_{0}^{* R}}{d \sigma_{1}^{* R}}<0$ which immediately implies $\frac{d p_{0}^{* R}}{d m}<0$. Therefore we must have $\frac{d \Delta_{p_{0}}}{d m}>0$ when $m>1$.

When $m<1$, or equivalently fire-sale happens in a delayed equilibrium, we have $\Delta_{p_{0}}=$ $\frac{\left|p_{0}^{* R}-p_{0}^{*}\right|}{p_{0}^{* R}}$. As shown above we have that $\frac{d p_{0}^{* R}}{d m}<0$. And this implies $\frac{d \Delta_{p_{0}}}{d m}<0$ when $m<1$.

To see the last part of results on $m$, note that $\frac{d|m-1|}{d m}>0$ when $m>1$ and $\frac{d|m-1|}{d m}<0$ when $m<1$. This immediately establishes $\frac{d \Delta_{p_{0}}}{d|m-1|}>0$ for all values of $m$.

Now consider a change in $\gamma$. First we look at the case where $m<1$. In this case, we have $\Delta_{p_{0}}=\frac{p_{0}^{* R}-p_{0}^{* D}}{p_{0}^{* R}}$. It's obvious that $\frac{d \sigma_{1}^{* R}}{d \gamma}<0$. By 20, we know that $\frac{d p_{0}^{* R}}{d \sigma_{1}^{* R}}<0$ and hence it 
immediately follows that $\frac{d p_{0}^{* R}}{d \gamma}>0$. To see the effect on $p_{0}^{* D}$, taking the total derivative with respect to $\gamma$ in 18 we have:

$$
\frac{d p_{0}^{* D}}{d \gamma}=\frac{(1-\alpha)\left(\delta_{1}-1\right)}{\delta_{0}}\left(-\alpha \gamma \sigma_{1}^{* D^{-(1+\alpha)}} \frac{d \sigma_{1}^{* D}}{d \gamma}+\sigma_{1}^{* D^{-\alpha}}\right)
$$

Next we take the total derivative with respect to $\gamma$ from both sides of 16 . This implies:

$$
\frac{(1-\alpha)\left(\delta_{1}-1\right)}{\delta_{0}}\left(-\alpha \gamma \sigma_{1}^{* D^{-(1+\alpha)}} \frac{d \sigma_{1}^{* D}}{d \gamma}+\sigma_{1}^{* D^{-\alpha}}\right)+\frac{\alpha\left(\delta_{1}-1\right)}{\delta_{1}}\left((1-\alpha) \gamma \sigma_{1}^{* D^{-\alpha}} \frac{d \sigma_{1}^{* D}}{d \gamma}+\sigma_{1}^{* D^{1-\alpha}}\right)=0
$$

The above equations together imply:

$$
\frac{d p_{0}^{* D}}{d \gamma}=-\frac{\alpha\left(\delta_{1}-1\right)}{\delta_{1}}\left((1-\alpha) \gamma \sigma_{1}^{* D^{-\alpha}} \frac{d \sigma_{1}^{* D}}{d \gamma}+\sigma_{1}^{* D^{1-\alpha}}\right)
$$

If $\frac{d \sigma_{1}^{* D}}{d \gamma}>0$, then all components in the parenthesis are strictly positive and therefore we have $\frac{d p_{0}^{* D}}{d \gamma}<0$. To see why $\frac{d \sigma_{1}^{* D}}{d \gamma}>0$, note that when $m<1$, we need to have $\sigma_{1}^{* D}<1$ and that the function in 16 is strictly decreasing in $\sigma_{1}^{* D}<1$. This implies that an increase in $\gamma$, which shifts the function in $16 \mathrm{up}$, leads to a higher value of $\sigma_{1}^{* D}$ in equilibrium. As $\frac{d p_{0}^{* R}}{d \gamma}>0$ and $\frac{d p_{0}^{* D}}{d \gamma}<0$, we have $\frac{d \Delta_{p_{0}}}{d \gamma}>0$ when $m<1$.

For the last part, consider a change in $\gamma$ when $m>\frac{\delta_{1}}{\delta_{0}}$. Since $\frac{\delta_{1}}{\delta_{0}}>1$, we have $\Delta_{p_{0}}=$ $\frac{p_{0}^{* D}-p_{0}^{* R}}{p_{0}^{* D}}$. We use the expression above for $\frac{d p_{0}^{* D}}{d \gamma}$ again:

$$
\frac{d p_{0}^{* D}}{d \gamma}=\frac{(1-\alpha)\left(\delta_{1}-1\right)}{\delta_{0}}\left(-\alpha \gamma \sigma_{1}^{* D^{-(1+\alpha)}} \frac{d \sigma_{1}^{* D}}{d \gamma}+\sigma_{1}^{* D^{-\alpha}}\right)
$$

Since $m>\frac{\delta_{1}}{\delta_{0}}$, we know that $\sigma_{1}^{* D}>m>\frac{\delta_{1}}{\delta_{0}}$. This implies that $\frac{d \sigma_{1}^{* D}}{d \gamma}<0$ since 16 is strictly increasing for all $\sigma_{1}^{* D}>\frac{\delta_{1}}{\delta_{0}}$, which combined with the above leads to $\frac{d p_{0}^{* D}}{d \gamma}>0$ as all terms in parenthesis are positive.

To $\operatorname{sign} \frac{d p_{0}^{* R}}{d \gamma}$, using 19 , we take the total derivative with respect to $\gamma$ which gives:

$$
\frac{d p_{0}^{* R}}{d \gamma}=\left(\frac{1}{\delta_{0}}-\frac{\sigma_{1}^{* R}}{\delta_{1}}\right)\left(1-\frac{\alpha \gamma}{\sigma_{1}^{* R}} \frac{d \sigma_{1}^{* R}}{d \gamma}\right) \sigma_{1}^{* R^{-\alpha}}-\frac{\gamma}{\sigma_{1}^{* R}} \frac{d \sigma_{1}^{* R}}{d \gamma} \sigma_{1}^{* R^{-\alpha}}
$$

Now using the expression for $\sigma_{1}^{* R}$ above we have:

$$
\frac{d \sigma_{1}^{* R}}{d \gamma}=\frac{(m-1) m^{1-\alpha}}{\left(1-\gamma m^{1-\alpha}\right)^{2}}
$$


Putting this back into the expression for $\frac{d p_{0}^{* R}}{d \gamma}$, we get:

$\frac{d p_{0}^{* R}}{d \gamma}=\left\{\left(\frac{\left(m-\gamma m^{1-\alpha}\right)\left(1-\gamma m^{1-\alpha}\right)}{\gamma m^{1-\alpha}(m-1)}-\alpha\right)\left(\frac{1}{\delta_{0}}-\frac{m-\gamma m^{1-\alpha}}{\left(1-\gamma m^{1-\alpha}\right) \delta_{1}}\right)-1\right\} \frac{(m-1) \gamma m^{1-\alpha}}{\left(1-\gamma m^{1-\alpha}\right)\left(m-\gamma m^{1-\alpha}\right)} \sigma_{1}^{* R^{-\alpha}}$

Therefore, if the term in the bracket is negative we have $\frac{d p_{0}^{* R}}{d \gamma}<0$. In this case, since we clearly have $\frac{d p_{0}^{* D}}{d \gamma}>0$, we get $\frac{d \Delta_{p_{0}}}{d \gamma}>0$. This ends the proof.

Proof of Lemma 5. To see this suppose $W_{j}-W_{i}=\Delta>0$. Let $\bar{U}_{0 j}^{B}-\bar{U}_{0 i}^{B}=\epsilon$ and $\bar{U}_{0 j}^{S}-\bar{U}_{0 i}^{S}=\eta$. If both $\epsilon$ and $\eta$ are positive, $j$ Pareto dominates $i$ and no transfers are needed. Suppose without loss of generality that $\epsilon>0$ but $\eta \leq 0$. We know by assumption that $\epsilon+\frac{\eta}{\delta_{0}} m=\Delta>0$. The planner can give a lump sum subsidy equal to $-\frac{\eta}{\delta_{0}}$ to the sellers while levy a tax equal to $-\frac{\eta}{\delta_{0}} m$ on buyers in equilibrium $j$ to achieve a new allocation $j^{\prime}$. Then we will have:

$$
\left\{\begin{array}{l}
\bar{U}_{0 j^{\prime}}^{S^{\prime}}=\bar{U}_{0 j}^{S}-\eta=\bar{U}_{0 i}^{S}, \\
\bar{U}_{0 j^{\prime}}^{B^{\prime}}=\bar{U}_{0 j}^{B}+\frac{\eta}{\delta_{0}} m=\left(\bar{U}_{0 i}^{B}+\epsilon\right)+\frac{\eta}{\delta_{0}} m=\bar{U}_{0 i}^{B}+\Delta>\bar{U}_{0 i}^{B}
\end{array}\right.
$$

Hence in the new allocation $j^{\prime}$, buyers are strictly better off and sellers are at least as well off. This implies that $j^{\prime}$ is Pareto superior to $i$.

Proof of Proposition 4. Using 9 and 27 we can rewrite the the welfare as a function of the (inverse of) market tightness at $t=1$ :

$$
\begin{array}{r}
W=\left\{\gamma m^{1-\alpha}+\frac{m-\gamma m^{1-\alpha}}{\delta_{0}}+\frac{(1-\alpha)\left(m-\gamma m^{1-\alpha}\right)\left(\delta_{1}-1\right)}{\delta_{0}} \gamma \sigma_{1}^{*-\alpha}+\right. \\
\left.\frac{\alpha\left(\delta_{1}-1\right)\left(1-\gamma m^{1-\alpha}\right)}{\delta_{1}} \gamma \sigma_{1}^{* 1-\alpha}\right\} d_{2}
\end{array}
$$

Taking the derivative of $W$ with respect to $\sigma_{1}^{*}$ gives:

$$
\frac{d W}{d \sigma_{1}^{*}}=\frac{\alpha(1-\alpha)\left(\delta_{1}-1\right)\left(1-\gamma m^{1-\alpha}\right)}{\delta_{1}} \gamma \sigma_{1}^{*-(1+\alpha)}\left\{\sigma_{1}^{*}-\frac{\delta_{1}}{\delta_{0}} \frac{m-\gamma m^{1-\alpha}}{1-\gamma m^{1-\alpha}}\right\} d_{2}
$$

It is evident that $W$ is strictly decreasing for $\sigma_{1}^{*}<\frac{\delta_{1}}{\delta_{0}} \frac{m-\gamma m^{1-\alpha}}{1-\gamma m^{1-\alpha}}$ and strictly increasing for $\sigma_{1}^{*}>\frac{\delta_{1}}{\delta_{0}} \frac{m-\gamma m^{1-\alpha}}{1-\gamma m^{1-\alpha}}$ and has a minimum at $\sigma_{1 m i n}^{*}=\frac{\delta_{1}}{\delta_{0}} \frac{m-\gamma m^{1-\alpha}}{1-\gamma m^{1-\alpha}}$.

When $m>1$, we have: 


$$
\sigma_{1}^{* D}<\sigma_{1}^{* R}=\frac{m-\gamma m^{1-\alpha}}{1-\gamma m^{1-\alpha}}<\sigma_{1 m i n}^{*}
$$

Where $\sigma_{1}^{* R}$ and $\sigma_{1}^{* D}$ are the (inverse of) market tightness at $t=1$ for the run and delayed equilibria. The last inequality follows from $\delta_{0}<\delta_{1}$. This implies that $W^{D}>W^{R}$. For the case of $m<1$, we know that $\sigma_{1}^{* R}=\frac{m-\gamma m^{1-\alpha}}{1-\gamma m^{1-\alpha}}<\sigma_{1}^{* D}<m$. If $m \leq \sigma_{1 m i n}^{*}$ we have $W^{R}>W^{D}$ because $W$ is strictly decreasing on the left of $\sigma_{1 m i n}^{*}$. We show that there is a threshold $0<\xi<1$ for which $m \leq \sigma_{1 m i n}^{*}$ as long as $m \geq \xi$. To this end define:

$$
\xi(m) \equiv \frac{m-\gamma m^{1-\alpha}}{1-\gamma m^{1-\alpha}} / m
$$

Taking derivative with respect to $m$ gives:

$$
\xi^{\prime}(m)=\left\{1-\left(\frac{\alpha \gamma m^{1-\alpha}-1}{\alpha \gamma m^{1-\alpha}}\right)\left(\frac{1-\gamma m^{-\alpha}}{m^{-1}-\gamma m^{-\alpha}}\right)\right\} \frac{\alpha \gamma m^{-(1+\alpha)}}{m^{-1}-\gamma m^{-\alpha}}
$$

We note that the first term above is always strictly positive and hence $\xi^{\prime}(m)>0$ and $\xi(m)$ is strictly increasing for all $\gamma^{\frac{1}{\alpha}}<m<1$. Hence we have:

$$
m \leq \frac{\delta_{1}}{\delta_{0}} \frac{m-\gamma m^{1-\alpha}}{1-\gamma m^{1-\alpha}} \Longleftrightarrow \xi(m) \geq \frac{\delta_{0}}{\delta_{1}} \Longleftrightarrow m \geq \xi^{-1}\left(\frac{\delta_{0}}{\delta_{1}}\right)
$$

We also note that $\xi\left(\gamma^{\frac{1}{\alpha}}\right)=0$ and $\xi(1)=1$ which implies that $\xi:\left[\gamma^{\frac{1}{\alpha}}, 1\right] \rightarrow[0,1]$ is a one-toone mapping and so it has an inverse on $[0,1]$. Hence given that $\frac{\delta_{0}}{\delta_{1}} \in[0,1]$ the threshold is $\xi=\xi^{-1}\left(\frac{\delta_{0}}{\delta_{1}}\right)<1$. This completes the proof. 